\title{
ÉLECTRICITÉ
}

\section{Le Calcul Pratique des Courants de Court-circuit entre Phases ${ }^{(1)}$}

\author{
(SUITE ET FIN)
}

\section{DEUXIEME PARTIE.}

\section{Applications \\ CHAPITRE I.}

Les Problèmes pratiques nécessitant le calcul du courant de c.c entre phases.

\section{1. - GENERALITES.}

Un appareil installé dans un circuit doit être prévu, non seulement pour tenir indéfiniment le courant normal, mais encore pour supporter le courant le plus intense qui peut passer dans le circuit, courant qui n'a aucune relation avec le courant normal. Ce counant exceptionnel influe considé rablement sur la conception, les dimensions, le prix de l'appareil. Il peut atteindre 5 à 10 fois le courant normal dans un alternateur, 10 à 40 fois le courant normal dans un transformateur, et plusieurs centaines de fois le courant normal dans un réducteur d'intensité.

Deux cas sont à distinguer :

$\left.1^{\circ}\right)$ appareils devant supporter le courant exceptionnel sans dommáge tant qu'aucune cause extérieure ne vient l'interrompre. Ce sera le cas des machines tournantes, des transformateurs, des réactances, des câbles, des réducteurs d'intensilé.

$2^{\circ}$ ) appareils d'interruption dont le rôle est de couper ce courant exceptionnel. Ce sera le cas des disjoncteurs, des fusibles.

Une surintensité se manifeste par des contraintes mécaniques et thermiques; le calcul de la valeur instantanée maximum du courant à l'instant initial, et de la courbe du courant efficace pendant les premières secondes, permet de déterminer ces contraintes.

\section{2. - CONTRAINTES MECANIQUES.}

Le courant ne peut être interrompu dans la partie crois_ sante avant qu'il ait atteint sa valeur instantanée maximum, que par les fusibles limiteurs que nous considérons par la suite ; dans tous les autres cas, le maximum est effectivement atteint.

La force électromagnétique appliquée aux conducteurs est maximum à l'instant initial, et décroît ensuite. La contrainte qui s'exerce s'ur les supports des conducteurs (pièces de maintien et de calage) n'est pas un choc instantané : la force agissante tend à déplacer le conducteur ; une contrainte se développe graduellement pendant la déformation. Dans ce temps le courant décroît, et l'effet résultant dépend de cette décroissance.
Lin calcul tenant compte de ces considérations peut être mené assez loin dans le cas simple de la déformation des jeux de barres des tableaux. Ce cas csl cependant déjà compliqué, car une barre encastrée possède une période propre de vibration qui peut entrer cn résonance avec la période de la variation du courant : les efforts sont alors considérablement accrus.

Dans le cas du réducteur d'intensité, la force électromagnétique qui tend à détruire l'appareil en cas de grande surintensité dépend du type d'appareil : avec le type barre cet. te force est nulle, il ne se pose aucun problème de déformation.

Mais ce modèle d'appareil n'est réalisable qu'à partir d'un courant de 80 à $100 \mathrm{~A}$; il n'est économique qu'à partir de $500 \mathrm{~A}$, aussi les appareils à primaire bobiné subsistent-ils pour les intensités modérées.

On réalise normalement deux classes d'appareils qui doivent résister aux conditions suivantes :

Classe 2 : la première amplitude est inférieure à $240 \mathrm{I}_{n}$

Classe 3 : la première amplitude est inférieure à $600 \mathrm{I}_{n}$

\section{3. - CONTRAINTES THERMIQUES.}

La contrainte thermique est un échauffement : une masse de cuivre soumise à des pertes Joule pendant le temps $t$, la chaleur produite se dépensant intégralcment à élever la température du cuivre (ce qui néglige toute déperdition extérieure par propagation, convection ou rayonnement : hypothèse admissible lorsque ( ne dépasse pas quelques secondes) atteint une élévation de température donnée par la relation :

$$
\Delta \Theta=0,5\left(\frac{\delta}{10}\right)^{2} t
$$

¿ ćtant la densilé de courant dans le cujvre.

(soit un échauffement d'un demi-degré par seconde pour 10 a mp. $\mathrm{mm}^{2}$ de densité).

Si l'on s'impose, par excmple, de ne pas dépasser pour la surintensité considérée un échauffement (s'ajoutant à la tempéráture préexistante du cuivre) de $75^{\circ}$, puis de $200^{\circ}$, on obtient respectivement les densités de courant limite de 120 et $180 \mathrm{amp}$. par $\mathrm{mm}^{2}$ pour unc durée de 1 seconde.

Un couranl de c.c. $I_{1}$ maintenu pendant le temps $t$ détermine le même échauffement que le courant $\mathrm{I}_{3} / \bar{l}$ pendant une scconde.

En réalité, pendant le court-circuit la densité ne reste pas constante ; il faut déterminer le courant efficace relatif à l'intervalle de temps que l'on considène sur la courbe du courant de c.c. en fonction du temps, avant d'appliquer la formule précédente.

Lo temps $t$ qui intervient dans les relations ci-dessus, est 
la durée maximum qui peut séparer l'apparition du c.c. de la coupure du c.c. par le disjoncteur; il dépend de la durée de temporisation du relais commandant le disjoncleur du feeder intéressé. On voit donc que, pour limiter les effets thermiques, il faut réduire cette lemporisation au minimum.

La valeur admissible pour $\Delta \Theta$ dépend du genre d'appareil considéré ; pour $\Delta \Theta$ supérieur à $200^{\circ}$ la résistance mécanique du Guivre diminue très rapidement.

Considérons quelques cas particuliers.

1. - Feeder souterrain.

La densité en régime normal est de l'ondre de 2 A par $\mathrm{mm}^{2}$. La température à ne dépasser en aucun cas pour la bonne conservation de l'isolant est environ $100^{\circ}$. On prendra done $\Delta \Theta=75^{\circ}$.

\section{2. - Bobine de réactance.}

Pour une réactance dans l'air, à refroidissement naturel, la condition d'échauffement et de pertes conduit à une densité en régime normal de 1,5 $\mathrm{A}$ environ (condition 1 ).

On impose en général de ne pas dépasser $200^{\circ}$ d'augmentation d'échauffement après 5 secondes, ce qui conduit à une densité limite de $90 \mathrm{~A}$ par $\mathrm{mm}^{2}$ (condition 2).

Application numérique :

\begin{tabular}{|c|c|c|c|c|c|}
\hline \multicolumn{3}{|c|}{ Réactance } & Courant & \multicolumn{2}{|c|}{$\begin{array}{c}\text { Section minimum } \\
\text { déterminé par }\end{array}$} \\
\hline $\begin{array}{c}\text { pour } \\
\text { cent }\end{array}$ & $\begin{array}{c}\text { tension } \\
\text { réseau }\end{array}$ & $\begin{array}{c}\text { courant } \\
\text { normal }\end{array}$ & $\begin{array}{c}\text { de c. c. } \\
\text { dendition } \\
1\end{array}$ & $\begin{array}{c}\text { condition } \\
2\end{array}$ \\
\hline 3 & $10 \mathrm{KV}$ & $250 \mathrm{~A}$ & 8200 & $91 \mathrm{~mm}^{2}$ & $167 \mathrm{~mm}^{2}$ \\
1 & 10 & 60 & 6000 & 67 & 40 \\
\hline
\end{tabular}

On voit que, dans le premier cas, la section à adopter est limitée par l'échauffement en régime normal, et dans le second cas, par l'échauffement en court-circuit.

\section{3. - Réducteur d'intensité.}

Un réducteur d'intensité doit en général tenir le courant de c.c. de la dérivation dans laquelle il se trouve, pendant plusieurs secondes. (En effet, on utilise souvent à l'heure actuelle les relais d'intensité à temps constants échelonnés pour doubler les productions instantanées des différents éléments du réseau par une protection de sécurité à maximum simple. L'échelonnement des relais conduit à différer jusqu'à 5 ou 6 secondes les relais les plus voisins du centre générateur).

Le courant de c.c. dans une dérivation, exprimé en multiple du courant normal de cette dérivation, est une fonction du temps d'abord décroissante, puis constante (la valeur constante étant atteinte après 1,5 seconde au voisinage de la centrale et $0,5 \mathrm{sec}$. environ en un point éloigné). (Pour un temps total de l'ordre de 5 à 6 secondes, la valeur efficace résultante se confond avec la valeur permanente).

Le courant de c.c. permanent à considérer est celui entre deux phases, qui est beaucoup plus élevé que le c.c. triphasé.

Le réducteur sera défini par :

$1^{\circ}$ le courant de c.c. constant équivalent (entre 2 phases) $I_{c c}$.

$2^{\circ}$ le temps $t$ pendant lequel dure ce c.c.

Désignons par $m$ le rapport du courant d'échauffement une seconde $I_{1} V \bar{t}$ au courant normal $I_{n}$.
La densité de courant limite pour une seconde étant 180 amp. mmª la densité normale sera

$$
\bar{s}_{n}=\frac{180}{m}
$$

On a réglementé deux classes d'appareils, à ce point de vue :

Appareils de classe 2 - correspondant à $m=80$.

Appareil de classe 3 - correspondant à $m=200$

La densité dans le conducteur primaire est alors :

$$
\begin{aligned}
& \delta_{n}=2,25 \text { pour la classe } 2 ; \\
& \delta_{n}=0,90 \text { pour la classe } 3 .
\end{aligned}
$$

La théorie du transformateur de mesure permet d'établir que la puissance de précision $\mathrm{P}$ d'un réducteur de classe de précision donnée est proportionnelle au carré du nombre d'ampères-tours du primaire pour le courant normal. Les ampères-tours que l'on peut loger dans un encombrement sont égaux à :

$$
\text { volume du cuivre } \times \delta_{n} \text {. }
$$

Il en résulte que $\mathrm{P}$ varie comme ${\delta_{n}}^{2}$, et par suite en raison inverse de $\mathbf{m}^{2}$.

Un appareil qui donne 30 VA pour $m=80$ donnera 4,8 VA pour $m=200$.

\section{4. - Appareils d'interruption.}

a) Disjoncteurs.

1. - Courant normal et courant exceptionnel.

L'appareil est étabi pour deux courants distincts :

$1^{\circ}$ Le counant normal de durée qui pose un premier problème de tenue des contacts, d'échauffement, de coupure ;

$2^{\circ}$ Ie courant exceptionnel momentané qui pose un problème beaucoup plus difficile de coupure.

2. - Eléments caractéristiques de la difficulté de coupure.

La difficulté de la coupure d'un circuit à courant alternatifs croit principalement avec les éléments suivants :

$1^{\circ}$ courant de rupture $I_{r}$;

$2^{\circ}$ tension de rétablissement $\mathrm{U}_{r}$;

$3^{\circ}$ taux de rétablissement de cette tension.

Précisons les notions :

Le courant de rupture $\mathrm{I}_{r}$ est par définition la valeur efficace de la composante alternative du courant à l'instant où les contacts commencent à se séparer. L'amplitude de la composante alternative à même valeur pour les 3 phases.

Pourquoi faut-il considérer pour caractériser la coupure la composante alternative et non le courant total ? Il y a 2 raisons :

a) parce qu'en général la rupture ne se produit qu'après le premier dixième de seconde, et la composante continue est alors très réduite ;

b) parce que la composante continue est nulle lorsque le décalage entre les valeurs instantanées du courant et de la tension est de $90^{\circ}$ (cas de la coupure d'un circuit très inductif). C'est pour ce décalage que la coupure est la plus difficile car la tension est maximum lorsque le courant passe par zéro et sa pleine valeur se dépense à réamorcer l'arc.

Quand le décalage est autre, il existe bien une composante continue qui majore le courant total, mais la tension est inférieure à son maximum lorsque le courant total s'annule. L'extinction de l'are peut donc être plus facile; bien que le 
courant total soit alors sensiblement plus élevé. La valeur du courant total ne peut donc être prise comme caractéristique de la difficulté de coupure ; elle ferait apparaître une valeur de courant de rupture illusoire.

La tension de rétablissement est par définition la valeur efficace de l'onde de tension qui s'établit immédiatement après l'extinction de l'arc, et qui tend à provoquer son réamorçage dans l'espace ionisé. Cette tension mesure la f.e.m. interne du générateur. Le courant de court-circuit, en grande partie réactif, exerce une action démagnétisante qui réduit d'autant plus le flux résultant de l'alternateur que la dunée du c.c. est plus grande, et que le c.c. est plus intense. La tension de rétablissement s'en trouve d'autant plus réduite. Sa valeur résulte de la variation de la composante alternative du courant de c.c. en fonction du temps. Le rapport de la tension de rétablissement à la tension de service est sensiblement égal au rapport de l'amplitude de la composante alternative lors de l'extinction de l'arc, à l'amplitude de la composante alternative initiale.

Le taux de rélablissement de la tension est mesuré par la tangente $\frac{d \mathrm{E}}{d t}$. Lorsque cette valeur est élevée, le temps pendant lequel la déionisation peut se produire est réduit, et le réamorçage de l'arc est facilité. La difficulté de la coupure augmente.

La valeur $\frac{d \mathrm{E}}{d t}$ est en général très élevée (de l'ordre des $\mathrm{KV}$ par microseconde). Cette valeur dépend essentiellement des caractéristiques du réseau ; elle croît avec la fréquence propre d'oscillation du réseau, qui est de la forme :

$$
\frac{1}{2 \pi \sqrt{\mathrm{LC}}}
$$

Cette fréquence propre est de l'ordre de quelques milliers de périodes par seconde ; sa mesure est très délicate à cause des harmoniques. Plus la capacité et la longueur du réseau sont importantes, plus la fréquence propre est faible et moins le taux de rétablissement est élevé.

Le problème est finalement de prédéterminer, pour un disjoncteur qui sera placé en un point d'un réseau, les valeurs $\mathrm{I}_{r}, \mathrm{U}_{r}$ et $\frac{d \mathrm{E}}{d t}$.

$1^{\circ} I_{r}$ peut être calculé assez exactement en fonction des conditions locales, comme il a été expliqué dans la première partie de cette étude.

$2^{\circ} \mathrm{U}_{r}$ a pour limite supérieure la tension de service du réseau U. Sa valeur résulte de la variation de la composante alternative pendant le c.c. comme il a été expliqué.

$3^{\circ}$ La valeur de $\frac{d \mathrm{E}}{d t}$ échappe encore à toute prédétermination et même à toute mesure précise ; aussi ne s'en occupet-on pas dans la pratique courante puisque c'est une grandeur dont l'exploitant ne peut avoir actuellement la perception.

On admet finalement que les éléments courants de rupture et tension de rétablissement, suffisent à caractériser le pouvoir de coupure de l'appareil ; on choisit souvent conventionnellement $\mathrm{U}_{r}$ égal à sa limite supérieure, et l'on dira que le disjoncteur sur un réseau triphasé, est capable de la puissance de coupure $\mathrm{UI}_{r} \sqrt{3}$ exprimée en KVA.

Il est clair, en vertu de ce qui précède, que ce n'est là qu'une règle simpliste qui n'entre plas dans la vraie complication du phénomène. (On a signalé le cas où l'emploi d'une réactance dans un circuit pour limiter $I_{r}$ avait eu pour effet d'augmenter le $\frac{d \mathrm{E}}{d t}$ et par suite la difficulté de coupure).

Toutefois, la règle du pouvoir de coupure répond aux exigences pratiques qui ne peuvent se préoccuper des circonstances sujeltes à varier lors des modifications de configura-

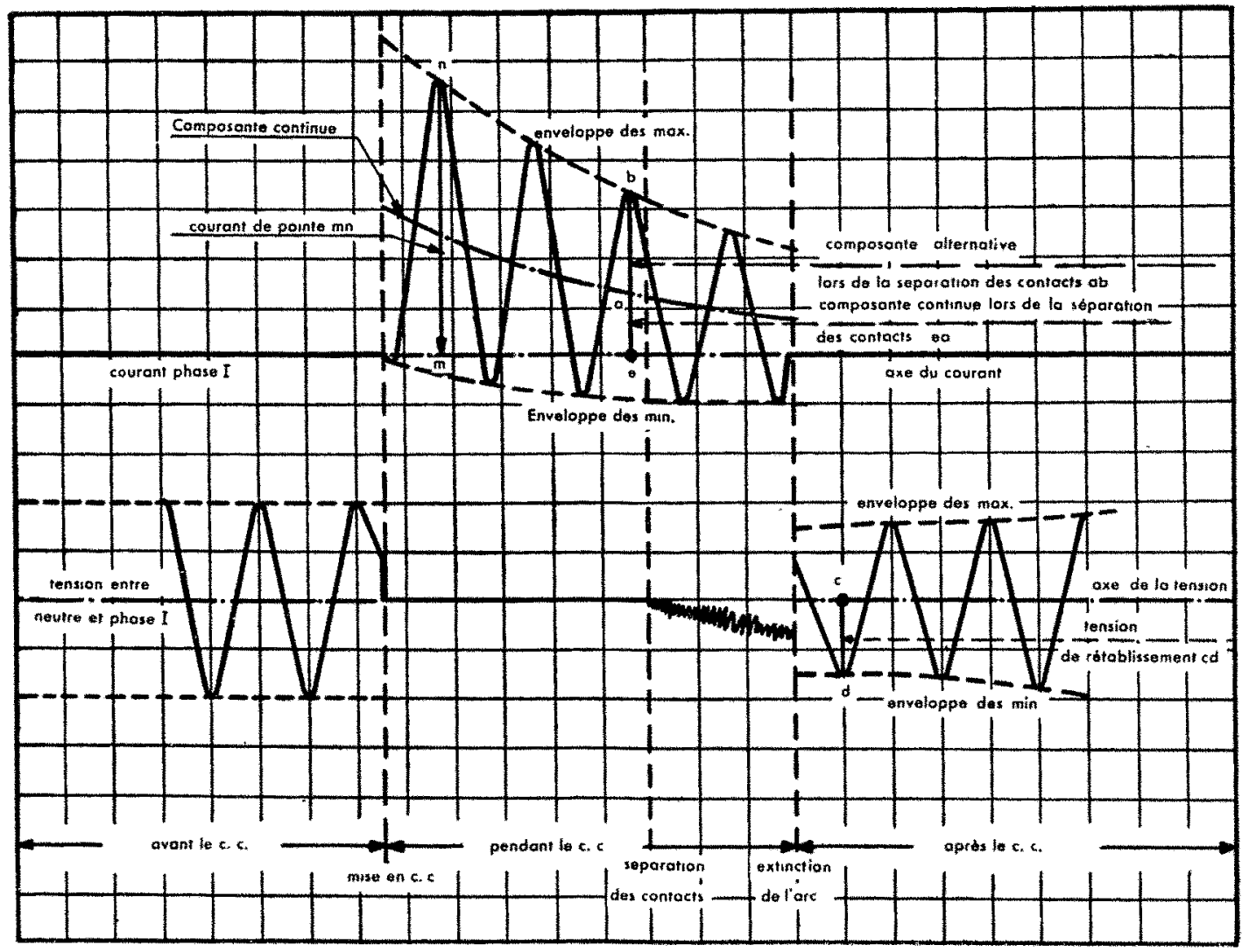

Fig. 30. 
tion du réseau. On se contente de calculer le courant de c.c. à l'endroit où sera placé l'appareil à l'instant où se produira la coupure.

\section{3. - Oscillogramme du courant et de la tensilon lors d'une} coupure (fig.30).

L'oscillogramme de courant donne la variation instantanée du courant pendant toute la durée du court-circuit ; il ne permet cependant pas de reconnaître l'instant où les contacts commencent à se séparer.

L'oscillogramme de tension donne la variation de la tension qui, sinusoïdale et constante avant le c.c., tombe à zéro pendant le c.c. franc. Lorsque les contacts commencent à se séparer, la tension augmente. La courbe de tension prend alors un aspect dentelé pendant la durée de l'arc. Lors de l'extinction de l'are la courbe de tension devient sinusoidale plus ou moins amortie selon les conditions relatives à l'alternateur. On trace l'enveloppe des maxima et des minima.

La valeur efficace de la tension de rétablissement est $\frac{c d}{\sqrt{2}}$

La puissance apparente de coupure est pour la phase considérée :

$$
\frac{a b}{\sqrt{2}} \times \frac{c d}{\sqrt{2}}
$$

Les deux valeurs dont on fait le produit correspondent donc à des instants différents - d'autant plus différents que l'arc est plus long à s'éteindre.

4. - Détermination du courant de rupture sur la courbe de la composante alternative du courant efficace de court-circuit.

Pour déterminer $I_{r}$ sur la courbe donnant le courant de court-circuit en fonction du temps, il convient de préciser l'inslant où les contacts commencent à se séparer :

le disjoncteur est maintenu fermé par une serrure. En général cette serrure est sous le contrôle d'un relais d'intensité : lorsque le courant dans le circuit principal dépasse une certaine valeur limite $I_{1}$, la serrure est déclenchée par le relais (direct ou indirect).

Entre l'instant où le courant passe en croissant par la valeur $I_{1}$ et celui où la serrure est déclenchée, il s'écoule un temps $\Delta t_{1}$, qui est en général réglable : c'est le retard $d u$ relais.

La serrure étint déclenchée, il s'écoule un temps $\Delta t_{2}$ avan! que les contacts ne commencent à se séparer, à cause des inerties mécaniques. Ce temps est habituellement compris entre 5 périodes (disjoncteurs modernes et rapides) et 15 périodes (disjoncteurs moins mapides).

L'abscisse à considérer sur la courbe d'intensité dans le circuit en fonction du temps pour déterminer la valeur du courant de rupture d'un disjoncteur est $\Delta t_{1}+\Delta t_{2}$.

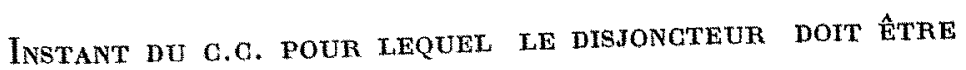
CALIBRE.

Un disjoncteur est appelé à couper le courant de courtcircuit à l'instant $t_{0}$ qui suit l'établissement du court-circuit. Comment choisir l'instant $t_{0}$ ? Ce choix ayant une importance essentielle sur la capacité de coupure, et par suite le prix de l'appareil.

Les réponses possibles sont les suivantes :

$1^{\mathrm{re}}$ Réponse : prendre $t_{0}=0$ (c'est-à-dire prévoir le dis- joncteur pour le courant initial, et une tension de rétablissement qui alors certainement très voisine de la tension normale).

La raison de ce choix $t_{0}=0$ est que, si le disjoncteur commence à couper pour un défaut peu important qui va en s'aggravant, le défaut peut s'affranchir au moment précis de l'ouverture des contacts. Lo disjoncteur coupe alors le courant initial : c'est le phénomène assez rare de la coupure prématurée.

$2^{\text {me }}$ Réponse : prendre $t_{0}=0,2 \mathrm{sec}$. ce temps correspondant sensiblement au retard mécanique de déclenchement des disjoncteurs alimentés par relais non différés.

$3^{\text {me }}$ Réponse : prendre $t=0,5$ à 2 sec. - ce temps dépendant du retard au déclenchement apporté par un relais différé.

La raison de différer les relais est :

$\left.1^{\circ}\right)$ réduction du courant, encore accentuée par le fait que les limiteurs d'intensité ont le temps de faire sentir leur action, et que la réaction d'induit a le temps d'intervenir pour réduire notablement la tension de rupture. Le disjoncteur devient beaucoup plus petit.

$2^{\circ}$ ) emploi d'une protection basée sur la sélection à temps constant en cascade, par un échelonnement de retards fixes allant en croissant à mesure que l'on s'éloigne du point de défaut pour se rapprocher du générateur ; le disjoncteur le plus voisin du générateur n'est pas alors plus chargé que celui voisin du défaut.

Le fait de différer le relais présente des inconvénients graves:

à cause des effets thermiques de la surintensité sur les réducteurs d'intensité, les câbles de petite section, et de l'aggravation des défauts sur les machines qui rend les réparations plus coûtéuses.

à cause de la stabilité des générateurs (décrochages des machines synchrones) ;

à cause de la perturbation introduite dans le réseau récepteur par la persistance d'un défaut (baisse de tension, etc...).

Ces inconvénients l'emportent de beaucoup sur les avantages, et la pratique moderne est de réaliser une protection sélective non aifférée basée sur la modification que les courants de défaut apportent aux éléments qui fixent l'état électrique du réseau (protection différentielle, etc...).

En conclusion, il intervient un élément d'appréciation dans le choix du temps $t_{0}$ pour lequel doit etre calibré le disjoncteur. Il conviendra en général de prendre $t_{0}=0,2 \mathrm{sec}$. de manière à réserver toutes les possibilités de réglage des relais.

\section{Court-circutt entre deux pHases.}

Le courant de c,c. entre 2 phases est en général, comme nous l'avons vu, un peu plus élevé après 0,2 seconde que le courant de c.c. triphasé. On calculera donc ce courant de préférence au courant de c.c. triphasé pour évaluer la capacité de coupure de l'appareil.

\section{5. - Puissances de coupure usuelles.}

Il est plus facile à réaliser des puissances de coupure élevées avec les disjoncteurs à très haute tension 150 à $220 \mathrm{KV}$ qu'avec les appareils à tension modérée $(10 \mathrm{KV})$ qui présentent des inerties plus grandes du fait des courants normaux généralement plus élevés, et des distances d'isolation moindres. 
A l'heure actuelle les puissances de coupure à $10 \mathrm{KV}$ ne dépassent pas couramment $500.000 \mathrm{KVA}$; tandis que pour 150 et $220 \mathrm{KV}$ on alteint 1 ct 2 millions de KVA.

Le prix de l'appareil $10 \mathrm{KV}$ suit la progression de la puissance de coupure ; tandis qu'à $150 \mathrm{KV}$ il n'y a pas d'économie sensible à réduire au-dessous d'une certaine limite la puissance de coupure (aussi le calcul de courants de c.c. dans ces réseaux ne nécessite-t-il pas une très grande précision).

\section{6. - Refermeture sur court-circuit.}

Lorsqu'on ferme un disjoncleur sur un court-circuit préexistant, les conditions diffèrent de celles de la coupure ordinaire : le courant initial atteint la valeur maximum du courant instantané total. Un disjoncteur doit pouvoir supporter ce courant de refermeture a moins que le circuit ne fasse l'objet d'un essai de mise sous tension progressive après coupure d'un c.c. pour s'assurer que le c.c. a disparu ; cependant, même dans ce cas, une fausse mancuvre reste possible). Le problème de refermeture sur c.c. conduit à des contraintes plus élevées que celui de la coupure au point de vue des efforts électrodynamiques. Une fermeture très rapide est nécessaire.

Remarque. - La coupure d'un courant intense entraîne loujours une certaine dégradation des contacts. Aussi un disjoncteur permettant une coupure correcte mais nécessitant alors un démontage pour la visite des pièces de contacts, n'est-il pas considéré comme satisfaisant. Les règlements actuels prescrivent que l'appareil doit supporter sans révision une coupure, suivie après une minute d'un réenclenchement sur c.c. aussitôt suivi de coupure, puis après une minute nouvel enclenchement et nouvelle coupure. Il va sans dire que ce régime est beaucoup plus difficile à tenir qu'une seule coupure correcte ; il ne sera d'ailleurs que très exceptionnellement réalisé en exploitation.

7. - Coupure dans lhuile et sans huile.

On a d'abord réalisé les appareils à coupure dans l'huile, l'huile étant le corps le plus apte à s'opposer à la réapparition de l'arc lors du rétablissement de la tension.

Ces appareils sont construits sans disposition spéciale jusqu'à $200.000 \mathrm{KVA}$, et avec chambre de rupture au-dessus. La chambre de rupture utilise la pression de l'huile vaporisée dans l'arc pour chasser l'huile entre les contacts et projeter la partie mobile. La coupure de l'arc est très rapide avec ces appareils (de l'ordre de la demi-période, tandis qu'il faut plusieurs périodes avec coupure ordinaire).

La technique du disjoncteur s'oriente à l'heure actuelle vers des appareils de conception différente :

$1^{\circ}$ - coupure dans l'eau, l'arc est soufflé par la vapeur d'eau volatilisée qui se détend. Ce type est dit à expansion, et convient pour les appareils du modele intérieur, les tensions ne dépassant par $25 \mathrm{KV}$ et les puissances de coupure 500 mille KVA.

$2^{\circ}$ - volume d'huile réduit (ne dépassant pas $1 \%$ du volume d'huile d'un disjoncteur ordinaire). Un jet d'huile perpendiculaire à l'arc vient le souffler, suivant le principe de la chambre de rupture. Ce type convient pour les appareils extérieurs, aux tensions élevées et grandes puissanices de coupure.

Ces appareils présentent l'avantage commun de supprimer le danger d'incendie, le risque d'explosion en cas d'avarie, et de permettre l'accès aux contacts (facilité de vérification de l'état des contacts après coupure d'un courant intense).
Un disjomcteur de ce genve ne demande donc pas autanl de marge sur la puissance de coupure qu'un appareil à huile du type coumant.

A l'heure actuelle pour les installations nouvelles, la pratique consiste à employer le disjoncteur sans huile dans les locaux où la place est très mesurée et où las conséquences d'une explosion, ou d'un incendie, seraient désastreuses (aggloménations).

Les idisjoncteurs à huile de capacité de coupure adéquate sont installés dans des cellules ouvertes sur l'extérieur, le couvercle et les bornes de l'appareil étant seuls accessibles de l'intérieur du bâtiment, et convenablement cloisonnés.

Sans doute l'emploi d'un disjoncteur à huile comporte-t-il un risque dès l'instant où l'explosion est considérée comme possible. Risque très comparable à celui qu'acceptent en général sans protester les personnes qui sont assises dans une salle juste au-dessous d'un lustre.

\section{Station d'EssaIs.}

La nécessité d'étudier systématiquement le rôle des différentes variables qui intéressent le phénomène très complexe de la coupure a conduit les constructeurs spécialisés à réaliser des stations d'essais permettant la mise au point de leur matériel.

Le problème se pose alors de savoir jusqu'a quel point un essai en station d'essais se rapproche des conditions de la coupure qui se rencontrent en service.

\section{$\left.1^{\circ}\right)$ Courant de rupture.}

On reproduit à l'essai la valeur du courant désiré lors de la séparation des contacts, mais en général avec une allure de décroissance différente de celle du réseau, car on provoque la coupure quelques périodes après avoir établi le c.c. (c'est-à-dire beaucoup plus tôt que lors de la coupure en service) de manière à bénéficier des premières amplitudes avant qu'elles ne soient amorties par le régime transitoire.

La composante continue n'aura donc pas nécessairement la même valeur qu'en service ; mais il est admis qu'elle n'influe pas appréciablement sur les conditions de la coupure. La composante continue sera d'ailleurs en général pratiquement disparue lors de l'extinction définitive de l'arc. Certains dispositifs d'essai permettent de la supprimer sur les 3 phases.

\section{$\left.2^{\circ}\right)$ Tension de rétablissement.}

On règle l'essai de manière à obtenir une tension de rétablissement égale à toute valeur désirée ; cette valeur est habituellement prise égale à la tension de service du réseau, ce qui est plus sévère que la réalité pour un disjoncteur placé au voisinage d'une centrale dans un réseau peu interconnecté.

$3^{\circ}$ ) Facteur de puissance du circuit coupé voisin de zéro ce qui est en général plus sévère que la réalité.

$\left.4^{\circ}\right)$ Fréquence propre du circuit d'essai plus élevée en général que celle d'un réseau, car les canalisations sont très courtes et la réactance de l'́ltternateur est très réduite ; la fréquence propre est de l'ordre de 30.000 périodes alons que dans un réseau clle ne dépasse souvent pas quelques milliers de périodes. Cela conduit à une valeur de $\frac{d \mathrm{E}}{d t}$ beaucoup plus élevée qu'en exploitation. 
$\left.5^{\circ}\right)$ Cycle de déclenchements et enclenchements successifs qui ne sera que très exceptionnellement réalisé en exploitation.

On voit en conclusion que l'essai, en station d'essais, est à différents égards plus dur que la coupure en service. Ainsi intervient-i] un élément d'appréciation lors du choix d'un disjoncteur : si la capacité de coupure nominale est garantie par un essai en plateforme, l'exploitant qui a calculé la capacilé de coupure requise, pourra être fondé d'adopter un apparcil de puissance nominale inférieure à la valeur limite calculée.

\section{b) Fusibles haute tenston.}

1. - Rôle du fusible.

Le fusible s'emploie sur feeders en dérivation : (fig. 31 ).

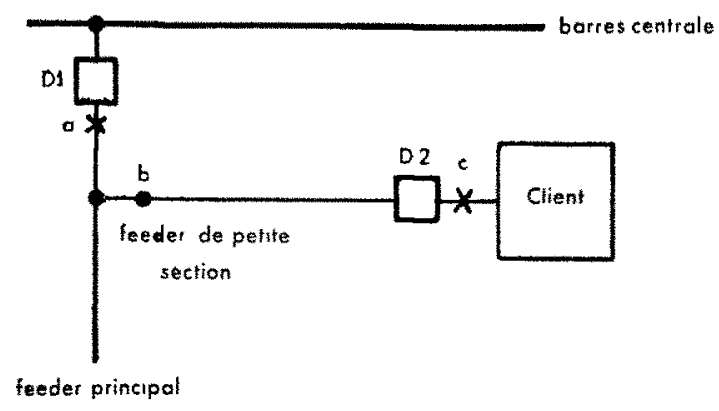

$1^{\circ}$ pour protéger les disjoncteurs $D_{1}$ ou $D_{2}$ insuffisants en plaçant le fusible en $a$ ou en $c$.

$2^{\circ}$ pour protéger un câble de petite section $b$ c en plaçant le fusible en $b$ ou en $c$, si la durée d'action du disjoncteux: $D_{1}$ est asson grande el la section du càble assez petite pour qu'en cas de défaut sur le cable celui-ci puisse s'endommager avant le déclenchement de $D_{1}$.

Si l'on place des fusibles simultanément en $a$ et en c, calibrés en rapport avec. l'intensité nominale en ces points, il n'y a pas de sélection certaine ; a peut couper en même temps que $c$ pour un défaut client. Il n'est donc pas logique de mettre un fusible en $a$, sauf pour parer momentanément à l'insuffisance d'un disjoncteur.

\section{2. - Galibre.}

Un fusible est caractérisé par son calibre, ou intensitế $I_{\varepsilon}$ la plus élevée qu'il puisse supporter indéfiniment sans fondre. On choisit $I_{c}$ supérieur au courant normal $I_{n}$ pour laisser passer les surcharges.

\section{3. - Courbe de fusion.}

La courbe du courant de fusion du fusible en fonction du temps est bien définie, figure 32 .

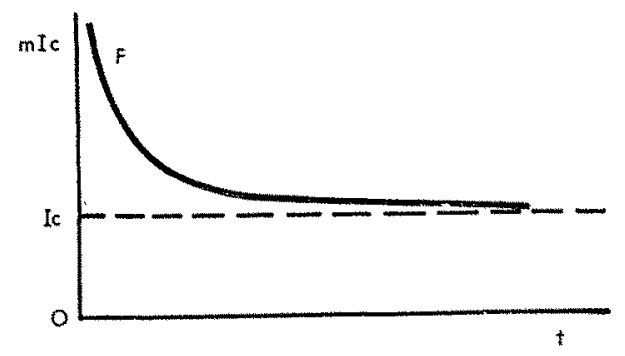

Fig. 32
Pour $I=I_{c}$ le fusible fond pour $t$ infini (asymptote).

Pour un multiple $m \mathrm{I}_{c}$ de $\mathrm{I}_{c}$, la durée est d'autant plus petite que $m$ est plus grand. Lorsque $m$ dépasse 25 , ce qui est toujours le cas pour un fusible placé en un point voisin du jeu de barres d'une centrale importante, le fusible coupe dans le premier septième de seconde.

Nous supposerons cette condition réalisée.

\section{4. - Les deux genres de fusibles.}

$I^{\text {er }}$ genre fusible-disjoncteur. - Le fusible coupe à la manière d'un disjoncteur, lorsque le courant passe par zéro. Ces appareils se construisent pour des calibres qui peuvent atteindre quelques centaines d'ampères.

$2^{\text {mo }}$ genre fusible-limiteur. - Le fusible interrompt le circuit dans la partie croissante de la courbe du courant de c.c. avant qu'il ait atteint sa pleine valeur, ce qui est avantageux pour limiter à une valeur très faible les efforts électrodynamiques en cas de c.c.

Ces fusibles ne se construisent que pour des intensités atteignant quelques dizaines d'ampères.

\section{5. - Capacité de coupure.}

Un fusible qui fonctionne dans le premier centième de seconde est caractérisé par son courant de pointe limite $\mathrm{I}_{\mathbf{l}}$. Ce courant est effectivement atteint avec un fusible-disjoncteur. Avec fusible-limiteur, ce courant de pointe est le courant qui serait atteint en remplaçant le fusible par une impédance nulle. Lorsqu'on prévoit l'utilisation d'un fusible en un point donné, où la valeur efficace initiale de la composante symétrique est $I_{0}$, la première pointe de courant peut atteindre la valeur $2,5 \mathrm{I}_{0}$; on choisira donc :

$$
2,5 \mathrm{I}_{0}<\mathrm{I}_{l} \text {. }
$$

\section{CHAPITRE II}

\section{Les Applications wsuelles}

\section{1. - GENERALITES.}

Nous nous proposons d'appliquer la méthode générale de calcul qui vient d'être exposée aux cas particuliers usuels, ce qui nous permettra d'avoir immédiatement des idées en présence des problèmes de court-circuit que pose un réseau.

Nous considérerons successivement les catégories de problè. mes les plus courants, pour des systèmes non bouclés.

\section{II. - LE GALGULL DU COURANT DE COURT-GIRCUIT AUX BARRES D'UNE GENTRALE.}

Soit une centrale $10.500 \mathrm{~V}$. dont la puissance peut être comprise entre 20.000 et $150.000 \mathrm{kVA}$ selon le nombre des unités en service (turbo-alternateurs).

On demande les réactances de court-circuit en ohms et les courants de court-circuit $I_{1}, I_{2}, I_{3}$, la centrale étant en charge ? 


\begin{tabular}{|c|c|c|c|c|c|c|c|c|c|c|c|}
\hline \multirow{2}{*}{$\mathbf{P}_{n}$} & \multirow{2}{*}{$\mathbf{I}_{n}$} & \multicolumn{3}{|c|}{ Réactance (ohms) } & \multicolumn{3}{|c|}{$\begin{array}{l}\text { Court-circuit triph. } \\
\text { (courant efficace symétrique) }\end{array}$} & \multicolumn{4}{|c|}{ C. C. (entre 2 phases) } \\
\hline & & $X_{f}$ & $X_{f}^{\prime}$ & $\mathbf{X}$ & $I_{1}$ & $\mathbf{I}_{2}$ & $\mathbf{I}_{3}$ & $X_{i}$ & $\mathrm{I}_{2}$ & $I_{1}$ & $I_{3}$ \\
\hline 20.000 & 1.100 & 0,66 & 1,04 & 12 & 9.200 & 5.800 & 1.500 & 0,66 & 8.000 & 6.200 & 2.500 \\
\hline 40.000 & 2.200 & 0,33 & 0,52 & 6 & 18.400 & 11.600 & 3.000 & 0,33 & 16.000 & 12.500 & 5.000 \\
\hline 60.000 & 3.300 & 0,22 & 0,35 & 4 & 27.500 & 17.400 & 4.500 & 0,22 & 24.000 & 18.500 & 7.500 \\
\hline 80000 & 4.400 & 0,165 & 0,26 & 3 & 36.800 & 23.200 & 6.000 & 0,165 & 32.000 & 25.000 & 10.000 \\
\hline 100.000 & 5.500 & 0,13 & 0,21 & 2,4 & 46.000 & 29.000 & 7.500 & 0,13 & 40.000 & 31.000 & 12.500 \\
\hline 150.000 & 8.300 & 0,087 & 0,14 & 1,6 & 69.000 & 43.000 & 11.200 & 0,087 & 60.000 & 46.500 & 18.700 \\
\hline
\end{tabular}

La puissance de coupure pour des disjoncteurs installés aux barres de la centrale prendront les valeurs suivantes, dans le cas de c. c. triphasé, selon l'intervalle de temps $\Delta t$ dont la coupure est différée $(1,2$ ou 5 dixièmes de seconde):

\begin{tabular}{|c|c|c|c|}
\hline \multirow{2}{*}{$P_{n}$} & \multicolumn{3}{|c|}{ Retard au déclenchement } \\
\cline { 2 - 4 } & 0,1 & 0,2 & \multicolumn{1}{c|}{0,5} \\
\hline 20.000 & 105000 & 75.000 & 45.000 \\
40.000 & 210.000 & 150.000 & 90.000 \\
60.000 & 315.000 & 225.000 & 135.000 \\
80.000 & 420.000 & 300.000 & 180.000 \\
100.000 & 520.000 & 375.000 & 225.000 \\
150.000 & 780.000 & 560.000 & 340.000 \\
\hline
\end{tabular}

(Ces valeurs résultent du tracé des courbes de courant de c. c. en fonction du temps, passant par les points $I_{1} I_{2} I_{3}$ ).

On voit combien le retard au déclenchement influe sur la capacité de colupure.

\section{Remarque :}

Pour une coupure à 0,2 seconde, he rapport du courant de c. c. initial (valeur symétrique) au colurant de c. c. lors de la coupure est 2,25.

Par suite, cette réduction portant sur la tension de rétubissement, les puissances de coupure vraies seront finalement :

\begin{tabular}{|c|c|}
\hline $\mathbf{P}_{n}$ & $\begin{array}{c}\text { Puissance de coupure } \\
\text { vraie }\end{array}$ \\
\hline 20.000 & 33.000 \\
40.000 & 67.000 \\
60.000 & 100.000 \\
80.000 & 134.000 \\
100.000 & 166.000 \\
150.000 & 250.000 \\
\hline
\end{tabular}

\section{III. - COURT-CIRCUIT DE FEEDER. - REACTANCE DE PROTECTION}

a) Cas d'un réseau infini : rôle d'une réactance de protection.

Considérons un réseau infini représenté par la barre $m n$, et une dérivation $D$ de courant normal $\mathbf{I}_{n}$ prise sur ce réseau par l'intermédiaire d'une réactance de valeur $S_{0}$ (fig. 33).

Le courant de c. c. dans la dérivation est infini pour lun c. c. en $a$; il prend, pour un c. c. en $b$, la valeur $I_{c c}$ donnée par la relation :

$$
\frac{\mathrm{I}_{c e}}{\mathrm{I}_{n}}=\frac{100}{\mathrm{~S}_{e}}
$$

Donc, avec une réactance de valeur $S_{\epsilon}$ en pour cent, le courant $I_{e e}$ passe d'une valeur infinie à la fraction $\frac{100}{S_{c}}$ de $I_{n}$.

On peut dire également qu'on limite la puissance apparente de c. c. $\mathrm{P}_{\mathrm{c}} \mathrm{a} \frac{100}{\mathrm{~S}_{e}}$ de la puissance apparente normale $\mathrm{P}_{n}$.

Exemple : Soit une dérivation de 100 amp. sur un réseau 10. $\mathrm{kV}$. On demande la réactance à disposer en tềe de cette

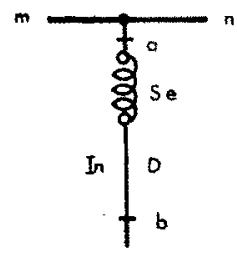

Fig. 33

dérivation pour limiter la puissance apparente du court-circuit dans cette dérivation à $100.000 \mathrm{kVA}$.

$$
\begin{aligned}
& \mathrm{I}_{n}=100 \mathrm{amp} . \\
& \mathrm{P}_{n}=100 \times 10 \times V=1730 \mathrm{kVA} . \\
& \frac{\mathrm{S}_{e}}{100}=\frac{1730}{100.000} \\
& \mathrm{~S}_{e}=1,73 \text { pour cent. } \\
& \mathrm{X}_{e}=\frac{1,73}{100} \frac{10.000}{\sqrt{3} \times 100}=1 \mathrm{ohm} .
\end{aligned}
$$

Application: Protection d'un câble issu des barres $m n$, par l'emploi d'une réactance.

La densité de courant en c. c. sera avec une densité normale de 2 A par $\mathrm{mm}^{2}$ dans le câble :

$$
2 \frac{100}{S_{e}}
$$

elle atteindra :

120 pour $\mathrm{S}_{s}=1,7 \%$. Le câble (voir page 40 ) peut tenir celte densité pendant 1 seconde.

85 pour $\mathrm{S}_{e}=2,4 \%$. Le câble (voir p. 40 ) peul tenir celte densité pendant 2 secondes.

53 pour $\mathrm{S}_{e}=3,8 \%$. Le câble (voir p. 40) peut tenir cette densité pendant 5 secondes.

En conclusion, il existe une relation entre la réactance cn \% el le temps pendant lequel le câble peut tenir le c.c. sur réseau infini.

On voit qu'une réactance de quelques pour cent permet de tenir quelques secondes : l'ordre de grandeur de la réactance est inmmédiatement connu.

b) Cas d'une centrale de puissance finie. - Réactance de protection.

Considérons les barres d'une centrale que nous schématiserons par un seul alternateur fictif A capable de la puissance 


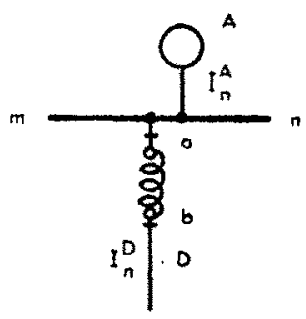

Fig. 34

génératrice totalisée de la tension $U$ et du courant normal $I_{n}^{A}$. (fig. 34).

Considérons une dérivation $D$ branchée sur le jeu des barres.

Soit $\mathrm{I}_{n}^{\mathrm{D}}$ le courant normal dans cette dérivation, qui sera la fraction $\frac{1}{m}$ de $\mathrm{I}_{n}^{A}$.

Pour un court-circuit en $a$ dans le jeu de barres ou dans son voisinage immédiat, le rapport du courant de c. c. au courant normal de l'alternateur n'est pas infini ; il est limité par la réactance $x$ de l'alternateur.

Supposons maintenant un court-circuit en $b$ (en aval de la réactance).

$s$ étant la réactance de l'alternateur en pour cent, rapportée à son courant normal $\mathrm{I}_{n}^{A}$.

$S_{e}$ la réactance $a b$ en pour cent rapportée (comme il est habiluel) au courant normal de la dérivation $\mathrm{I}_{n}^{\mathrm{D}}$.

$\mathrm{S}_{c}^{\prime}$ la réactance $a b$ rapportée à $\mathrm{I}_{n}^{\mathrm{A}}$.

On aura :

$$
\mathrm{S}_{e}^{\prime}=m \mathrm{~S}_{e} .
$$

La réactance totale qui intervient en c. c. est $\mathrm{s}+\mathrm{S}^{\prime}=\mathrm{s}$ $+m \mathrm{~S}_{c}$.

On aura:

$$
\frac{\mathrm{I}_{c e}^{b}}{\mathrm{I}_{n}^{\mathrm{D}}}=\frac{100}{\mathrm{~S}_{e}+\frac{s}{m}}
$$

La réactance de l'alternateur intervient d'autant moins pour réduire le courant de c. c. que la dérivation est plus petite.

\section{CAS D'UNE RÉaCTANCE EXTÉRIEURE ÉLEVÉE.}

Lorsque la réactance $S_{e}$ est grande (de l'ordre de la moitié de la réactance synchrone) lo courant dans la réactance peut dépasser la valeur qu'il prendrait si cette réactance était connoctée aux bornes d'un réseau infini parce que la tension aux bornes peut alors dépasser la valeur de la tension normale.

En effet :

la tension aux bornes de la réactance $\mathrm{S}_{e}$ est :

$$
\mathrm{U} \frac{\mathbf{S}_{\varepsilon}}{100} \frac{\mathbf{I}_{c c}}{\mathbf{I}_{n}}
$$

elle dépasse la tension normale pour :

$$
\frac{\mathrm{I}_{c c}}{\mathrm{I}_{n}}>\frac{100}{\mathrm{~S}_{e}}
$$

celle condition peut s'écrire :

$$
\frac{100}{\mathrm{~S}+\mathrm{S}_{e}} \frac{\mathrm{AT}_{i}}{\mathrm{AT}_{v}}>\frac{100}{\mathrm{~S}_{e}}
$$

Prenons: $\frac{\mathrm{AT}_{\mathrm{i}}}{\mathrm{AT}_{\mathrm{v}}}=3$

la relation s'écrit :

$$
\mathrm{S}_{e}>\frac{\mathrm{S}}{2}
$$

Le courant dans la réactance $S_{\varepsilon}$ dépassera dans ce cas le courant que l'on aurait dans une dérivation connectée aux bornes d'un réseau infini. Les relations algébriques ci-dessus ne s'appliquent d'ailleurs plus en pareil cas à cause de la saturation ; le courant de court-circuit doit se calculer par points.

Remarque : il peut arriver que la valeur permanente du courant de c. c. dépasse la valeur initiale. En effet :

valeur initiale $\frac{100}{s_{f}+\mathrm{S}_{e}}$

valeur permanente $\frac{100}{\mathrm{~S}+\mathrm{S}_{e}} \frac{\mathrm{AT}_{2}}{\mathrm{AT}_{v}}$

Supposons $\frac{\mathrm{AT}_{i}}{\mathrm{AT}_{v}}=3$ (ce qui correspond à une excitation voisine de l'excitation de pleine charge).

La valeur permanenle dêpasse la valeur initiale pour :

$$
\mathrm{S}_{e}>\frac{\mathrm{S}-3 s_{f}}{2}
$$

Application numérique.

Un turbo-alternateur $20.000 \mathrm{~kW} \cos \varphi=0,7$ à $10.500 \mathrm{~V}$. débite sur un feeder $\mathrm{D}$ de $170 \mathrm{kVA}$ protégé par une réactance $2 \%$. Un court-circuit se déclare en $\mathrm{C}$.

En supposant que le régulateur de tension établisse à ce moment l'excitation de pleine charge de l'alternateur, quel sera le colurant de c. c. permanent dans la réactance?

Données : Réactance synchrone 208 pour cent. Courant d'excitation de pleine charge 390 A. Excitation pour la tension normale prise sur la partie droite de la caractéristique à vide $123 \mathrm{~A}$.

Réactance de fuites : 19,5 pour cent (soit 0,76 ) ; AT de réaction d'induit au courant normal : 78.000 ; les AT inducteurs pour $390 \mathrm{~A}: 129.000$. Courbe de magnétisation $\mathrm{ATE}=f$ (E) (fig. 35).

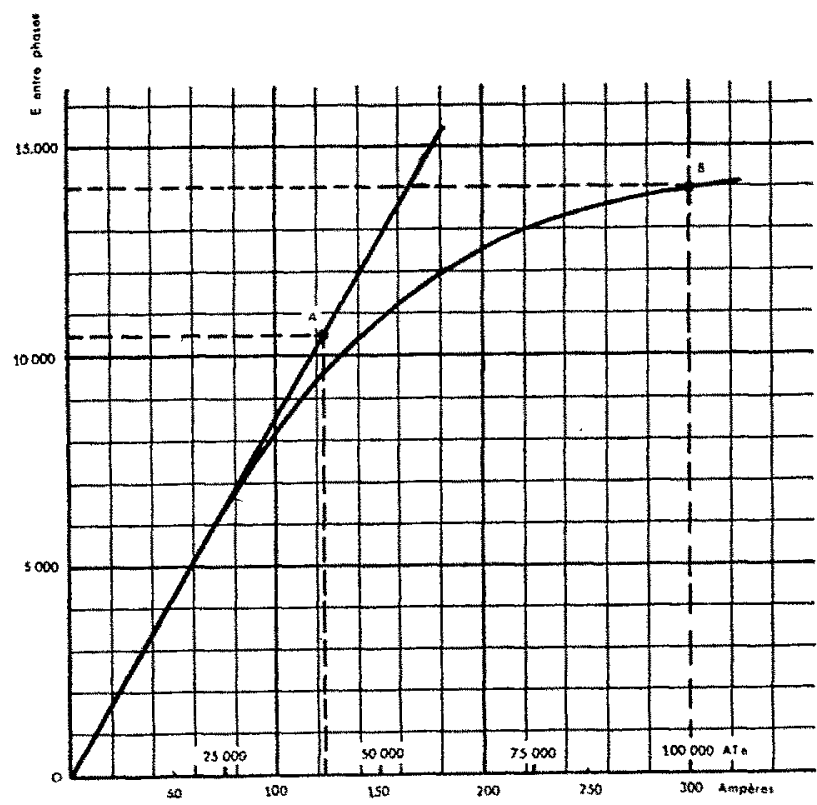

Fig: 35

Solution :

$$
\frac{20.000}{0,7}=28.600 \mathrm{kVA} \text {. }
$$

Courant normal $\left\{\begin{array}{l}\text { alternateur }: I_{n}=\frac{28.600}{10,5 \sqrt{3}}=1580 \\ \text { feeder } \frac{170}{10,5 \sqrt{3}}=9,5 \mathrm{~A} .\end{array}\right.$ 
Réactance extérieure $\frac{2}{100}=\frac{\mathrm{X}_{e} 9,5}{6100}$

$$
\text { d'où } \mathrm{X}_{\epsilon}=13 \text { ohms. }
$$

Ráactance extérieure en pour cent du courant normall de l'alternateur :

$13 \frac{1580 \times 100}{6100}=340$ pour cent.

D'où $I_{c c}=\frac{100}{208+340} \quad \frac{390}{123} \quad 1580=925 \mathrm{~A}$.

Comme $925 \times 13 \sqrt{3}=21.000$ volts on voil que le calcul est inexact à cause de la saturation.

Le calcul exact se conduit de la manière survante : la tension aux bornes est, par phase :

$$
\mathrm{U}=13 \mathrm{I}
$$

la réactance extérieure étant égale à 13 ohms.

la f.e.m. est :

$$
\mathrm{E}=13,76 \mathrm{I}
$$

(13,76 étant la somme de la réactance extérieure el de la réactance de fuites).

d'où sur la courbe de magnétisation

$$
\mathrm{AT}_{e}=f(13,76 \mathrm{I})
$$

Le nombre de spires de l'inducteur est :

$$
\frac{129.000}{390}=330
$$

D'autre part :

$$
\mathrm{AT}_{a}=\frac{78.000}{1.580} \quad \mathrm{I}=50 \mathrm{I}
$$

On aura donc d'après le diagramme de Potier :

$$
50 \mathrm{I}+f(13,76 \mathrm{I})=129.000
$$

Cette relation détermine $I=620 \mathrm{amp}$., soit 65 fois le courant normal dans la réactance; on sera en $B$ sur la courbe de magnétisation. La tension aux bornes sera $62013 \mathrm{~V}^{-} 3$ $=14.000$ Volts.

Application : Protection d'une dérivation de faible section voisine d'une centrale.

Une centrale de puissance apparente comprise entre 50.000 et $100.000 \mathrm{kVA}$ alimente directement un jeu de barres $10 \mathrm{kV}$.

Des départs en câble de $30 \mathrm{~mm}^{2}$ sont connectés à ce jeu de barres. Chacun est protégé par son disjoncteur D qui coupe

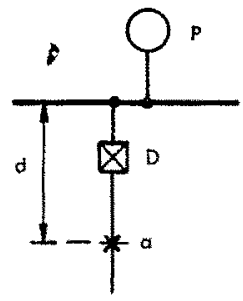

Fig. 36

en cas de c. c. instantané, dans un temps réglable entre 0,2 et $0,5 \mathrm{sec}$. Un défaut se produit sur le câble en $a$ à la distance $d$ de la centrale (fig. 36 ).

\section{Problème. 1.}

$d$ est voisin de zéro (défaut au voisinage de la centrale), on demande l'échauffement du câble :

a) après 0,5 sec. ?

b) après un centième de seconde ?

(Remarquons ici que, dans le cas du c. c. symétrique, la première demi-période dure $1 / 100$ seconde. Lorsque la com- posante continue est max. le premier max, est atteint après $1 / 100$ sec. environ car l'alternance est à compter sur l'axe moyen de la sinusoïde (fig. 4).

Nous calculerons l'échauffement du câble :

$1^{\circ}$ avec la composante continue maximum ;

$2^{\circ}$ avec la composante continue nulle ;

cn déterminant le courant efficace constant équivalent.

On suppose que les réactances subtransitoire et transitoire sont égales aux valeurs normales pour lurbo-alternateurs.

Composante alternative initiale (valeur efficace).

Courant efficace constant équivalent.

pour
0,5
sec. $\left\{\begin{array}{c}\text { a) avec composante } \\ \text { continue max. sans composante } \\ \text { continue. }\end{array}\right.$

pour (a) avec composante

$1 / 100$ b) sans compasante continue.

\begin{tabular}{|r|r|r|}
\hline 50.000 & 75.000 & 100.000 \\
\hline 24.000 & 36.000 & 48.000 \\
& & \\
17.000 & 25.500 & 34.000 \\
13.500 & 20.000 & 27.000 \\
35.000 & 52.000 & 70.000 \\
24.000 & 36.000 & 48.000 \\
\hline
\end{tabular}

Echauffement du câble après 0,5 seconde :

\begin{tabular}{l|l|l|l|} 
avec composante continue max. & $800^{\circ}$ & fusion & fusion \\
sans composante continue. & $500^{\circ}$ & fusion & tusion
\end{tabular}

Echauffement atteint après un centième de seconde :

avec composante continue max.

sans composante continue.

\begin{tabular}{r|r}
$70^{\circ}$ & $150^{\circ}$ \\
$30^{\circ}$ & $70^{\circ}$
\end{tabular}

$280^{\circ}$
$120^{\circ}$

Remarque :

Dans le cas d'un càble $125 \mathrm{~mm}^{2}$ avec retard 0,5 seconde on aunait :

Echauffement du câble :

\begin{tabular}{l|r|r|r} 
avec composante continue max. & $50^{\circ}$ & $120^{\circ}$ & $200^{\circ}$ \\
sans composante continue. & $30^{\circ}$ & $70^{\circ}$ & $120^{\circ}$
\end{tabular}

On voit donc quavec une centrale de $100.000 \mathrm{kVA}$ on arrive à la limite. On ne peut donc se permethe de différer la protection des feeders normaux au voisinag'e d'unc contrale de celte importance.

P hOBLÈME 2.

Le disjoncteur $\mathrm{D}$ étant de $100.000 \mathrm{kVA}$ on demande à partir de quelle distance $d$ le disjoncteur $D$ réglé à 0,2 sec. n'aura pas plus de $100.000 \mathrm{kVA}$ à couper?

Le calcul donne :

$$
d=
$$

$$
1500 \mathrm{~m} .1600 \mathrm{~m}
$$

$1700 \mathrm{~m}$.

\section{Problème 3.}

d est voisin de zéro. On demande quelle est la réactance inécessaire à insérer au dópart du feeder :

a) pour que le câble ne dépasse pas $75^{\circ}$ d'échauffement supiplémentaire en 0,5 seconde.

b) pour que le disjoncteur ne coupe pas plus de $100.000 \mathrm{kVA}$ en 0,2 sec.

Le calcul donne:

l Zéactance nécessaire en ohms

a) pour le cable

b) pour que le disjoncteur ne coupe pas plus de 100,000

kVA en $0,2 \mathrm{sec}$.

\begin{tabular}{|l|l|l|}
1 & 1,1 & 1,2 \\
0,6 & 0,76 & 0,85
\end{tabular} \mid


Pour achever de définir la réactance il faut préciser son courant normal :

Une dérivation $30 \mathrm{~mm}^{2}$ peut êtse utilisée à des courants nominaux :

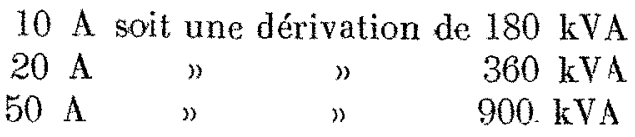

(ceci suppose que la section minimum admise dans le réseau est $30 \mathrm{~mm}^{2}$ ).

On aura les valeurs suivantes en \% des réactances nécessaires pour le câble :

$$
\begin{aligned}
& \text { dérivation de } 10 \wedge \\
& \text { " } 20 \\
& 20 \mathrm{~A} \\
& \text { ) } \\
& 50 \mathrm{~A} \\
& \begin{array}{l|l|}
0,165 \% & 0,18 \% \\
0,33 \% & 0,36 \% \\
0,825 \% & 0,905 \%
\end{array} \\
& 0,2 \% \\
& 0,4 \% \\
& 0,99 \%
\end{aligned}
$$

(Si on utilise une réactance communc à $n$ départs la réactance en \% est multipliée par $n$ ).

\section{Problème 4.}

$d$ est voisin de zéro. On demande :

a) Si on peut utiliser un fusible pour protéger le câble ?

b) sinon, quelle réactance il faut prévoir en mểme temps que le fusible?

Le calcul donne pour valeur efficace de la première demiondo du courant de c. c. symétrique :

$$
\begin{array}{l|r|r|r}
\text { en ampères } & 24.000 & 36.000 & 48,000 \\
\text { cn kVA } & 135.000 & 655.000 & 870.000
\end{array}
$$

Nous supposerons que l'on utilise un fusible ne permettant pas de dépasser un courant de c. c. dont la valeur efficace de la promière demi-onde symétrique est :

\begin{tabular}{c|c} 
ampères & $\mathrm{kVA}$ sous $10 \mathrm{KV}$ \\
\hline 15.000 & 260.000
\end{tabular}

Pour ne pas dépasser la limite ci-dessus il faut installer des réaclances définies comme suit en ohms :

$$
\begin{array}{l|l|l}
0,15 & 0,23 & 0,28
\end{array}
$$

Si on admet que le fusible coupe en 1/100 sec. l'échauffement atteint par le câble $30 \mathrm{~mm}^{2}$ seră :

cn cas d'onde symétrique : $13^{\circ}$,

en cats d'onde dissymétrique : environ $50^{\circ}$

donc le câble cst protégé.

\section{Problìme 5.}

Un réducteur d'intensité est installé sur un déparl $30 \mathrm{~mm}^{2}$ de $360 \mathrm{kVA}$.

On demande comment définir le réducteur ?

Le courant normal est $20 \mathrm{~A}$.

Le courant de c. c. cfficace constant équivalent pour $0,5 \mathrm{sec}$. a été calculé problème 1 .

Le rapport de surintensité du réducteur (voir p. 41) est, compte tenu de la composante continue maximum :

$$
\begin{aligned}
m=\frac{17.000}{20} & =850 \\
\frac{25.500}{20} & =1275 \\
\frac{34.000}{20} & =1700
\end{aligned}
$$

La densité de courant à adopter dans le bobinage primaire au régime normal sera :

$$
\frac{180}{8500,5}=0,30 \mathrm{amp} / \mathrm{mm}^{2} \quad 0,20 \quad 0,15
$$

au lieu de la densité de 0,9 qu'aurait un appareil de classe 3 . Il faudra donc un appar eil capable de loger respectivement 3; 4,5 et 6 fois plus d'ampères-tours primaires qu'un appareil de classe 3 capable des VA requis.

(Remarque : sur un départ $125 \mathrm{~mm}^{2}$ de $3.500 \mathrm{kVA}$, courant normal $193 \mathrm{~A}$, le rapport de surintensité serait :

$$
m=87 \quad 132 \quad 176
$$

Il faut un appareil de classe 2 dans le premier cas et de classe 3 daris les deux derniers).

\section{IV. - COURT-CIRCUIT EN UN POLNT QUELCONQUE} D'UN RESEAU ALIMENTE PAR UNE CENTRALE.

Le courant qui 'intéresse un disjoncteur déterminé n'est pas toujours le courant total de défaut ; il dépend de la position de ce disjoncteur dans le réseau. On calculera l'impédance résultante comprise entre l'alternateur, et chacun des points de défaut; ct le courant qui passe alors dans la branche du circuit où sc trouve le disjoncteur considéré.

Exemple 1. - Une seule centrale C (fig. 37).

Le défaut peut ètre en $a, b$ ou $c$.

défaut cn $a^{\prime}: D_{1}$ coupe le courant de c. c. de $A_{1}$;

" $b: D_{1}$ coupe lo courant de c. c. de $A_{2}$ et $A_{3}$;

" $\quad c: \mathrm{D}_{2}$ coupe le courant de $\mathrm{A}_{1}, \mathrm{~A}_{2}$ et $\mathrm{A}_{3}$.

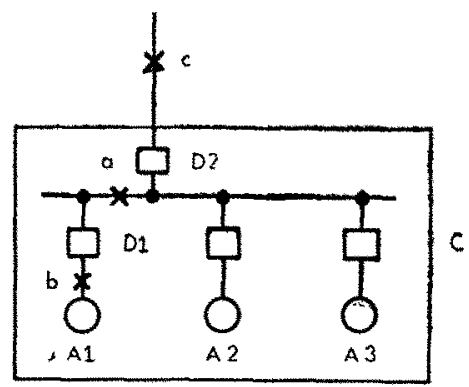

Fig. 37

\section{$1^{\circ}$ Application numérique.}

La centrale $\mathrm{C}$ est une centrale formée de 3 alternateurs de $25.000 \mathrm{KVA}$.

\begin{tabular}{|c|c|c|}
\hline $\begin{array}{c}\text { Endroit } \\
\text { du défaut }\end{array}$ & $\begin{array}{c}\text { Disjoncteur } \\
\text { intéressé }\end{array}$ & $\begin{array}{c}\text { Capacité de coupure } \\
\text { normale requise }\end{array}$ \\
\hline$a$ & $\mathrm{D}_{1}$ & 120.000 \\
$\mathrm{D}_{1}$ & $\mathrm{D}_{2}$ & 240.000 \\
& 360.000 \\
\hline
\end{tabular}

La capacité de coupure de ce tableau s'entend après $0,2 \mathrm{sec}$.

\section{V. - COURT-CIRGUIT DANS UN RESEAU ALIMENTE PAR INE GENTRALE FT UN FEEDER D'INTERCON -} NEXION.

Considérons une centrale qui reçoit sur ses barres un feeder d'interconnexion F (fig. 38) par lequel elle se trouve reliće à un réseau $R$ éloigné. 


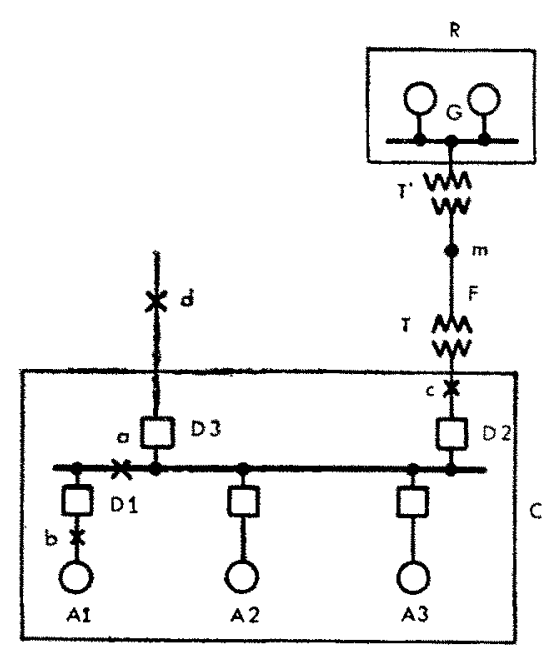

défaut en $a: \mathrm{D}_{1}$ coupe le c. c. de $\mathrm{A}_{1}$.

$» \quad b: D_{1}$ coupe le c. c. de $A_{2}$ et $A_{3}$ et celui de $F$.

$" \quad c: \mathrm{D}_{2}$ coupe le c. c. de $\mathrm{A}_{1}, \mathrm{~A}_{2}$ et $\mathrm{A}_{3}$.

b $\quad d: \mathrm{D}_{3}$ coupe le c. c. de $\mathrm{A}_{1}, \mathrm{~A}_{2}, \mathrm{~A}_{3}$ et $\mathrm{F}$.

Remarque, - La contribution du réseau $\mathrm{R}$ au courant de court-circuit qui apparaîl pour un défaut dans la zône d'action de la centrale $\mathrm{C}$, défaut coupé par $\mathrm{D}_{1}$ ou $\mathrm{D}_{3}$ dépend :

$1^{\circ}$ de l'impédance de la liaison $R-C$ reliant le réserau $R$ à la centrale $\mathrm{C}$.

$2^{\circ}$ de la puissance du réseau $R$.

\section{Rỏle de l'impédance.}

La liaison est en général une ligne à haute tension dont l'impédance tolalise celle de la ligne elle-même et des transfos $T^{T} T^{\prime}$ aux extrémités. Cette impédance élevée a pour effet de réduire sensiblement la contribution du réseau $R$.

Rôle de la puissance du réseau $R$.

Lorsque le réseau $\mathrm{R}$ devient de plus en plus puissant, la contribution du réseau $R$ s'accroit ; mais elle tend vers une limite, que l'on obtient en considérant que le réseau $R$ maintient une tension constante sur son jeu de barres. Cette romarque est importante : un réseau qui prévoit de s'interconnecter toujours davantage aveic d'autres, n'aura pas toujours à accroître la capacité de ses disjoncteurs. Il existe un plafond.

\section{Application numérique.}

Centrale $\mathrm{G}$-comme ci-dessus.

Centnale $\mathrm{R}$ - formée également de 3 alternateurs de $25.000 \mathrm{kVA}$.

Liaison R-C - tension $45 \mathrm{kV}$ - Longueur $10 \mathrm{~km}$, capable de $12.500 \mathrm{kVA}$.

\begin{tabular}{|c|c|c|}
\hline $\begin{array}{c}\text { Endroit } \\
\text { du défaut }\end{array}$ & $\begin{array}{c}\text { Disjoncteur } \\
\text { intéressé }\end{array}$ & $\begin{array}{c}\text { Capacité de coupure } \\
\text { normale requise }\end{array}$ \\
\hline$a \cdot$ & $\mathrm{D}_{1}$ & 120.000 \\
$b$ & $\mathrm{D}_{1}$ & 295.000 \\
$c$ & $\mathrm{D}_{2}$ & 360.000 \\
$d$ & $\mathrm{D}_{3}$ & 420.000 \\
\hline
\end{tabular}

Variante du cas précédent. - La liaison R-C est ainsi définie :

Tension $150 \mathrm{kV}$. - Longueur $60 \mathrm{~km}$. - Capable de $25.000 \mathrm{kVA}$.

\begin{tabular}{|c|c|c|}
\hline $\begin{array}{c}\text { Endroit } \\
\text { du défaut }\end{array}$ & $\begin{array}{c}\text { Disjoncteur } \\
\text { intéressé }\end{array}$ & $\begin{array}{c}\text { Capacité de coupure } \\
\text { normale requise }\end{array}$ \\
\hline$a$ & $\mathrm{D}_{1}$ & 120.000 \\
$b$ & $\mathrm{D}_{1}$ & 330.000 \\
$c$ & $\mathrm{D}_{2}$ & 360.000 \\
$d$ & $\mathrm{D}_{3}$ & 450.000 \\
\hline
\end{tabular}

Fariante du cas précédent. - Litison R-G $150 \mathrm{kV}$.

La centrale $R$ est un groupement très puissant qui maintient la tension constanto et égale a $150 \mathrm{kV}$ au point $m$. Lát liaison est capable de $25.000 \mathrm{kVA}$.

\begin{tabular}{|c|c|c|}
\hline $\begin{array}{c}\text { Endroit } \\
\text { du défaut }\end{array}$ & $\begin{array}{c}\text { Disjoncteur } \\
\text { intéressé }\end{array}$ & $\begin{array}{c}\text { Capacité de coupure } \\
\text { normale requise }\end{array}$ \\
\hline$a$ & $\mathrm{D}_{1}$ & 120.000 \\
$\mathrm{D}_{2}$ & 285.000 \\
$\mathrm{D}_{1}$ & $\mathrm{D}_{2}$ & 520.000 \\
$c$ & $\mathrm{D}_{3}$ & 360.000 \\
$c$ & 620.000 \\
\hline
\end{tabular}

Nous avons représenté fiqure 39 les puissances de coupure requises selon la variante considérée.

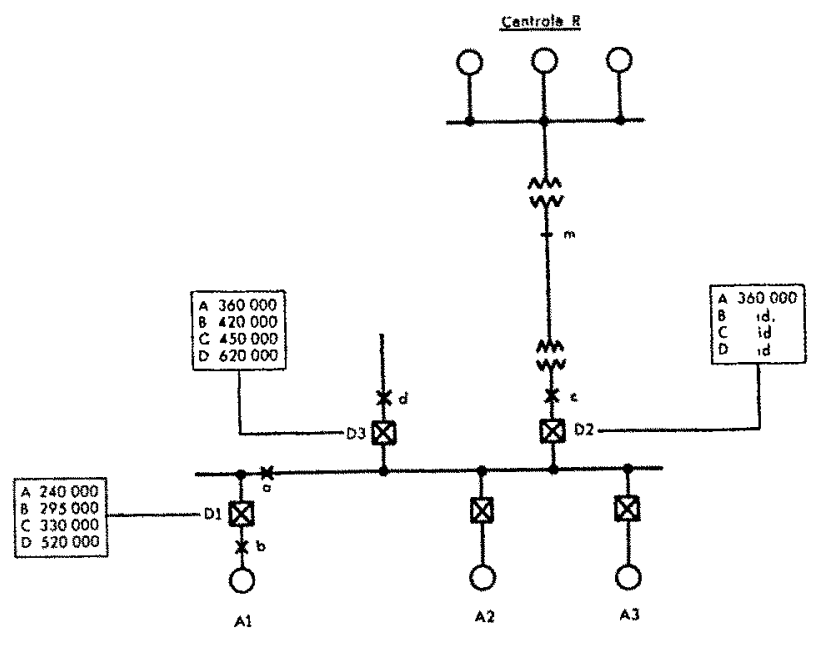

Centralo $C$

Fig. 39

LÉGENDE

\begin{tabular}{|c|c|c|c|}
\hline Varlante & Centrale $\mathrm{C}$ & Centrale $R$ & Liaison R-C \\
\hline $\begin{array}{l}\text { A } \\
B \\
C \\
D\end{array}$ & $\begin{array}{l}325000 \mathrm{kVA} \\
\text { id. } \\
\text { id. } \\
\text { id. }\end{array}$ & $\begin{array}{c}0 \\
3 \times 25000 \mathrm{kVA} \\
\text { id. } \\
\text { infinie }\end{array}$ & $\begin{array}{c}0 \\
12500 \mathrm{kVA}, 45 \mathrm{kV}, 10 \mathrm{~km} \\
25000 \mathrm{kVA}, 150 \mathrm{kV}, 60 \mathrm{~km} \\
\text { id. }\end{array}$ \\
\hline
\end{tabular}

VI. - COURT-CIRCUIT DANS UN RESEAU NON BOCCLE ALIMENTE PAR UN NOMBRE QUELGONQUE DE GENTRALES.

Le réscau comporte un certain nombre de centrales, reliécs entre elles par des lignes d'interconnexion. Le jeu de barres de chaque centrale totalise les puissances de c. o. de plusieurs générateurs. 


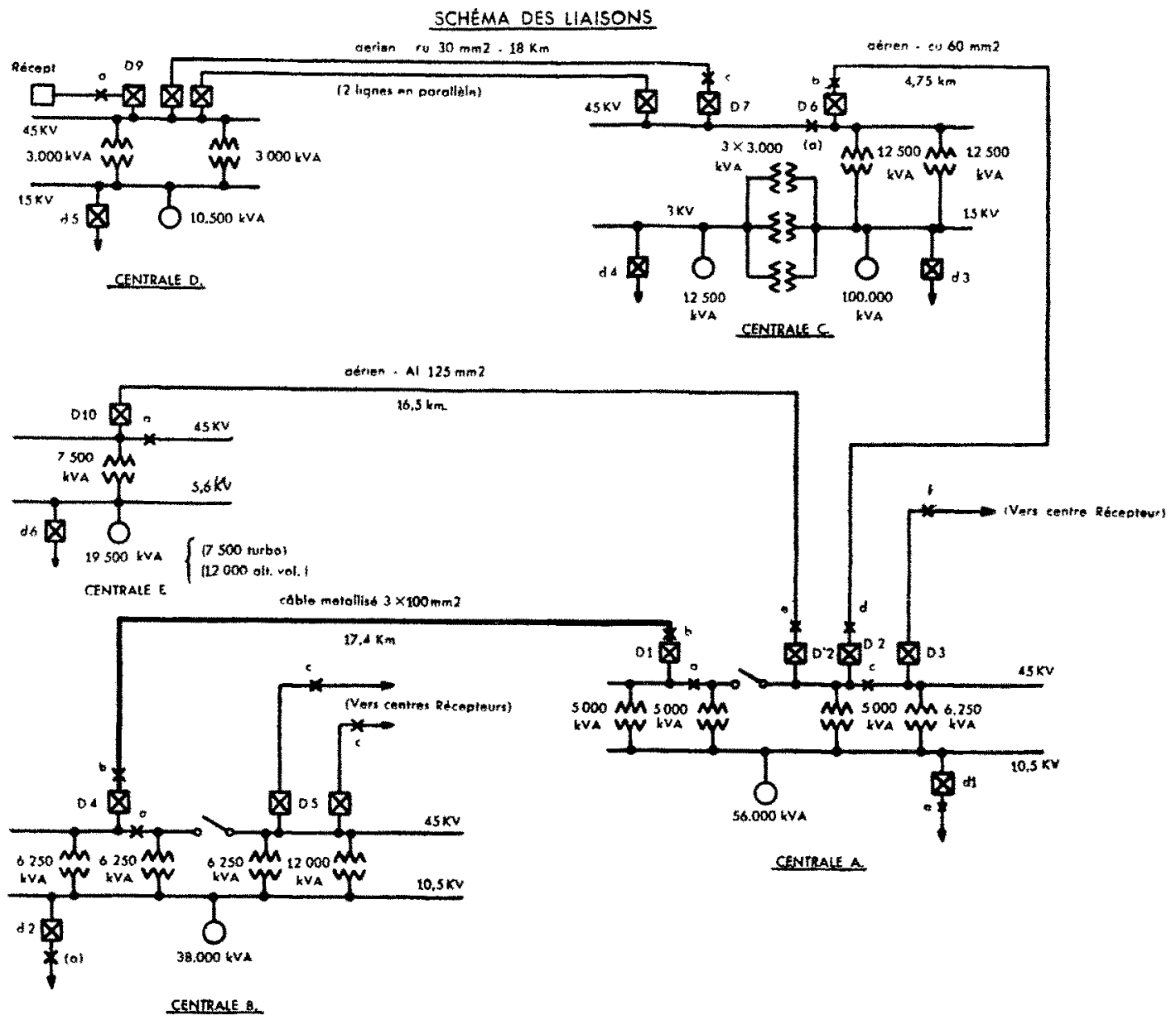

Fig. 40.

Le problème est de calculer le courant de c. c. sur une dérivation issue du jeu de barres de l'une quelconque des centrales.

L'exemple détaillé qui suit donnera une idée de la longueur des calculs qui sont nécessaires:

Un groupe de 5 centrales : A, B, C, D, E, sont interconnectées suivant le schéma figure 40 .

On se propose de calculer les puissances de coupure nécessaires. en cas de court-circuit triphasé pour les disjoncteurs $d_{1}, d_{2} \ldots \mathrm{D}_{1}, \mathrm{D}_{2} \ldots$ installés sur les départs $\mathrm{BT}$ et $\mathrm{HT}$ de chaque centrale après un temps de 0,2 seconde.

La figure 40 donne le schéma unifilaire des liaisons établies entre ces centrales ainsi que les caractéristiques de ces liaisons.

Les puissances des machines en service dans chaque centrale sont également indiquées. a) Valeur des impédances.

Pour effectuer les calculs des courants de c. c. nous adopterons la tension de base de $10,5 \mathrm{kV}$.

Les impédances des alternateurs, transformateurs et lignes doivent être calculées compte tenu de cette tension de base.

\section{1. - Alternateurs.}

a) Valeurs des impédances : (en \%). (Alternateurs à vide).

\begin{tabular}{|l|c|c|c|}
\hline & $\mathrm{Z}$ subtr. & $\mathrm{z}$ trans, & $\mathrm{z}$ permanent. \\
\cline { 2 - 4 } Turbo & $12 \%$ & $19 \%$ & $220 \%$ \\
Altern. Volants & $30 \%$ & $35 \%$ & $120 \%$ \\
\hline
\end{tabular}

b) Valeurs en ohms des impédlances pour les puissances en jeu dans les centrales et pour 10,5 kV.

\begin{tabular}{|c|c|c|c|c|c|c|c|}
\hline \multirow{2}{*}{ Genre de machine } & \multirow{2}{*}{$\mathrm{kVA}$} & \multicolumn{3}{|c|}{ Impédances å vide } & \multicolumn{3}{|c|}{$\begin{array}{l}\text { Impédances compte tenu de la charge } \\
\text { supposée a } \cos \phi=0,8\end{array}$} \\
\hline & & $Z$ subt. & $Z$ trans. & $Z$ perm. & $Z$ subt. & $Z$ trans. & $Z$ perm. \\
\hline \multirow[t]{6}{*}{ Turbos } & 7.500 & 1,75 & 2,77 & 32 & $1,6$. & 2,43 & $6.7+j 8,8$ \\
\hline & 10500 & 1,26 & 2 & 23 & 1,18 & 1,77 & $4,8+j 6,3$ \\
\hline & 12.500 & 1,06 & 1,68 & 19,5 & 0,98 & 1,47 & 4. $\quad+j 5,3$ \\
\hline & 38000 & 0,35 & 0,55 & 6,4 & 0,33 & 0,48 & $1,34+j 1,75$ \\
\hline & 56.000 & 0,24 & 0,37 & 4,3 & 0,22 & 0.32 & $0,9+j 1,17$ \\
\hline & 100.000 & 0,13 & 0,21 & 2,4 & 0,12 & 0,19 & $0,50+j 0,68$ \\
\hline Altern. volant & 12.000 & 2,77 & 3,25 & 11 & 2,2 & 2,6 & $2,7+j 4,8$ \\
\hline
\end{tabular}


Ces valeurs sont déterminées d'après les considérations exposée au chapitre " Court-circuit frane d'un alternateur en charge " et en supposant que les alternateurs fonctionnent sur réseau local, à leur pleine puissance et $\cos \varphi=0,8$.

La relation : $\frac{1}{z}=\frac{1}{Z}+\frac{1}{Z^{\prime}}$

$$
\begin{aligned}
& Z=\text { impédance des alterna- } \\
& \text { teurs à vide. } \\
& Z^{\prime}=\begin{array}{l}
\text { impédance équivalente } \\
\text { à la charge. }
\end{array} \\
& z=\begin{array}{l}
\text { impédance des alterna- } \\
\text { teurs en charge. }
\end{array}
\end{aligned}
$$

dans laquelle

permet de déterminer immédiatement, eu égard à l'hypothèse faite, les réactances en \% des alternateurs fonctionnant en charge,

Nous donnons ci-dessous le détail du calcul pour les turbos.

$$
\begin{aligned}
& Z^{\prime}=\frac{\mathrm{U}^{2}}{\mathrm{P}}(0,8+j 0,(i) \\
& \text { Z subtr. }=\frac{\mathrm{U}^{2}}{\mathrm{P}}(j 0,12) \quad \text { (P réseau losal }=\mathrm{P} \text { alternateurs) } \\
& \text { Z } \\
& Z \text { trans. }=\frac{\mathrm{U}^{2}}{\mathrm{P}}(j 0,19) \\
& \mathrm{Z} \text { perm. }=\frac{\mathrm{U}^{2}}{\mathrm{P}}\left(j^{2,2}\right) \\
& z \text { subtr. }=\frac{j^{0,12\left(0,8+j^{0,6)}\right.}}{0,8+j^{0,72}}=j 0,111=11,1 \%
\end{aligned}
$$

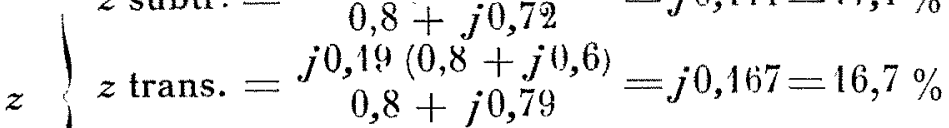

$$
\begin{aligned}
& z \text { perm. }=\frac{j^{2,2}\left(0,8+j^{0,6)}\right.}{0,8+j^{2,8}}=0,46+j^{0,6}
\end{aligned}
$$

\begin{tabular}{|c|c|c|c|}
\hline Centrale & $\begin{array}{c}\text { Puissance } \\
\mathrm{kVA}\end{array}$ & $\begin{array}{c}\text { Tension } \\
\text { de c. c. }\end{array}$ & $\begin{array}{c}\text { Réactance en } \\
\text { ohms sous } \\
10.5 \mathrm{kV}\end{array}$ \\
\hline $\mathrm{A}$ & $\begin{array}{c}5.000 \\
6.250\end{array}$ & $\begin{array}{c}6,5 \% \\
"\end{array}$ & $\begin{array}{c}1,44 \\
1,15\end{array}$ \\
\hline $\mathrm{B}$ & 6.250 & $4,4 \%$ & 0,77 \\
12.000 & $"$ & 0,4 \\
\hline $\mathrm{C}$ & 3.000 & $5 \%$ & 1,83 \\
& 12.500 & $6 \%$ & 0,53 \\
\hline $\mathrm{D}$ & 3.000 & $5 \%$ & 1,83 \\
\hline
\end{tabular}

III. - Lienes.

(Impédances ramenées à $10,5 \mathrm{kV}$ ).

\begin{tabular}{|c|c|c|c|}
\hline $\begin{array}{c}\text { Ligne } \\
\text { entre } \\
\text { centrales }\end{array}$ & Caractéristiques & $\begin{array}{c}\text { Long. } \\
\text { (Km) }\end{array}$ & Impédances \\
\hline $\mathrm{A}-\mathrm{B}$ & câble $3 \times 100 \mathrm{~mm}^{2}$ & 17,4 & $0,17+j 0,12$ \\
$\mathrm{~A}-\mathrm{C}$ & aérien $3 \times 60 \mathrm{~mm}^{2} \mathrm{Cu}$ & 4,75 & $0,07+j 0,1$ \\
$\mathrm{~A}-\mathrm{E}$ & aérien $3 \times 125, \mathrm{Al}$. & 16,5 & $0,21+j 0,36$ \\
$\mathrm{C}-\mathrm{D}$ & aérien $2(3 \times 30) \mathrm{Cu}$ & 18 & $0,29+j 0,20$ \\
\hline
\end{tabular}

b) Schémas des Impédances.

La puissance de coupure des disjoncteurs devant être calculée après un temps égal à 0,2 seconde, il est nécessaire pour détcrminer sa valeur de tracer la courbe du courant de c. c. en fonction du temps.

Ceci nous conduit à considérer les valeurs subtransitoires, transitoires et permanentes des impédances des alternateurs et, par conséquent, à établir 3 schémas pour les impédances.

Ces schémas sont représentés figure $41 a, b$, et $c$.

lls reproduisent le schéma unifilaire du système après avoir remplacé les alternateurs, transformateurs et lignes, par leurs impédances équivalentes.

Considérons par exemple Je schéma des impédances transitoires.

$$
\begin{aligned}
& \text { Turbo-alternateurs : } \mathrm{P}=56.000 \mathrm{kVA} . \mathrm{Z} \text { trans. }=j 0,32
\end{aligned}
$$

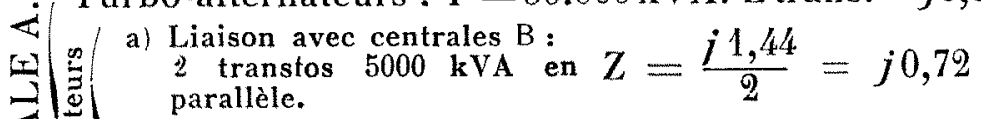

$$
\begin{aligned}
& Z=\frac{j 1,44 \times j 1,15}{j(1,44+1,15)}= \\
& =j^{0,64} \text {. }
\end{aligned}
$$

Turbo-alternateurs : $\mathrm{P}=38.000 \mathrm{kVA} . Z$ trans $=j 0,48$

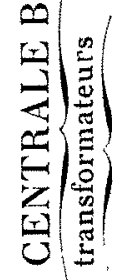
a) Liaison avec centrale $\mathrm{A}: \mathrm{kVA}$ en $\mathrm{Z}=\frac{j 0,77}{2}=j^{0,38}$
2 transfos $6250 \mathrm{kVA}$ parallèle.

b) Départ vers récepteurs :

1 transfo $6250 \mathrm{kVA}$ en parallèle avec 1 transfo $12.000 \mathrm{kVA}$.

$$
\begin{aligned}
Z & =\frac{j^{0,77 \times j 0,4}}{j 0,77+0,4}= \\
& =j 0,26 .
\end{aligned}
$$

Ligne entre centrales $\mathrm{A}-\mathrm{B} \quad \mathrm{Z}=0,17+j 0,12$

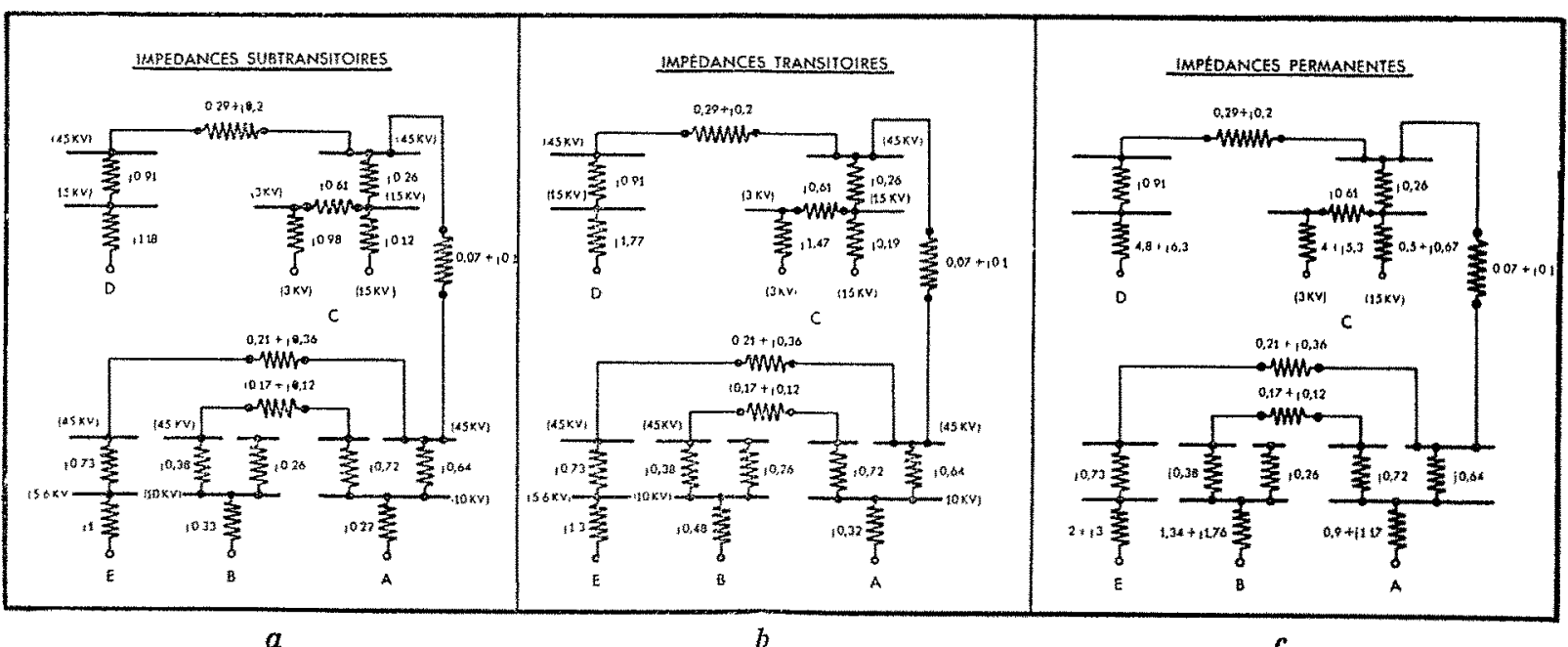

Fig. 41. 
REDUCTIONS SUCCESSIVES DES IMPEDANCES SUBTRANSITOIRES

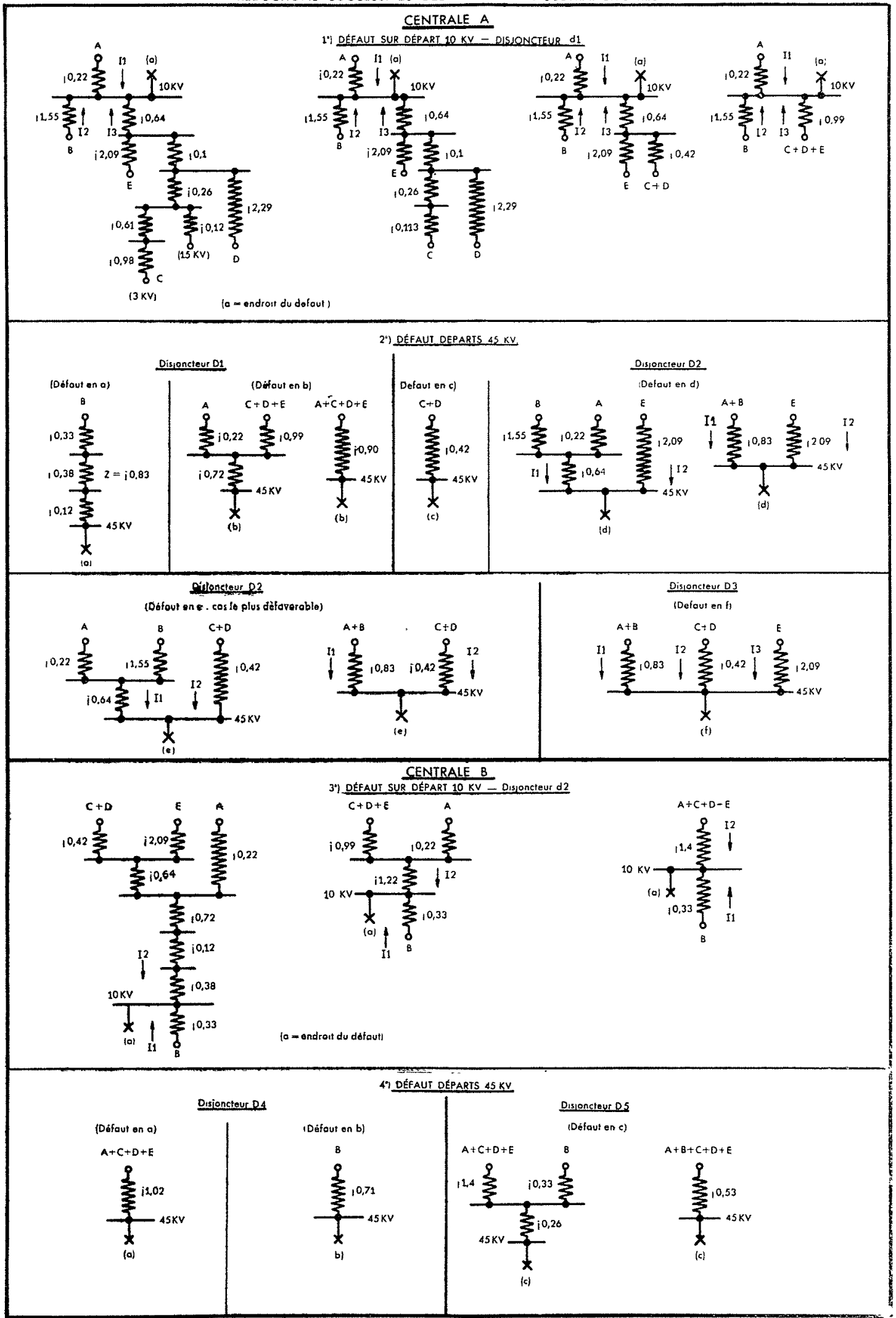

Fig. 42. 


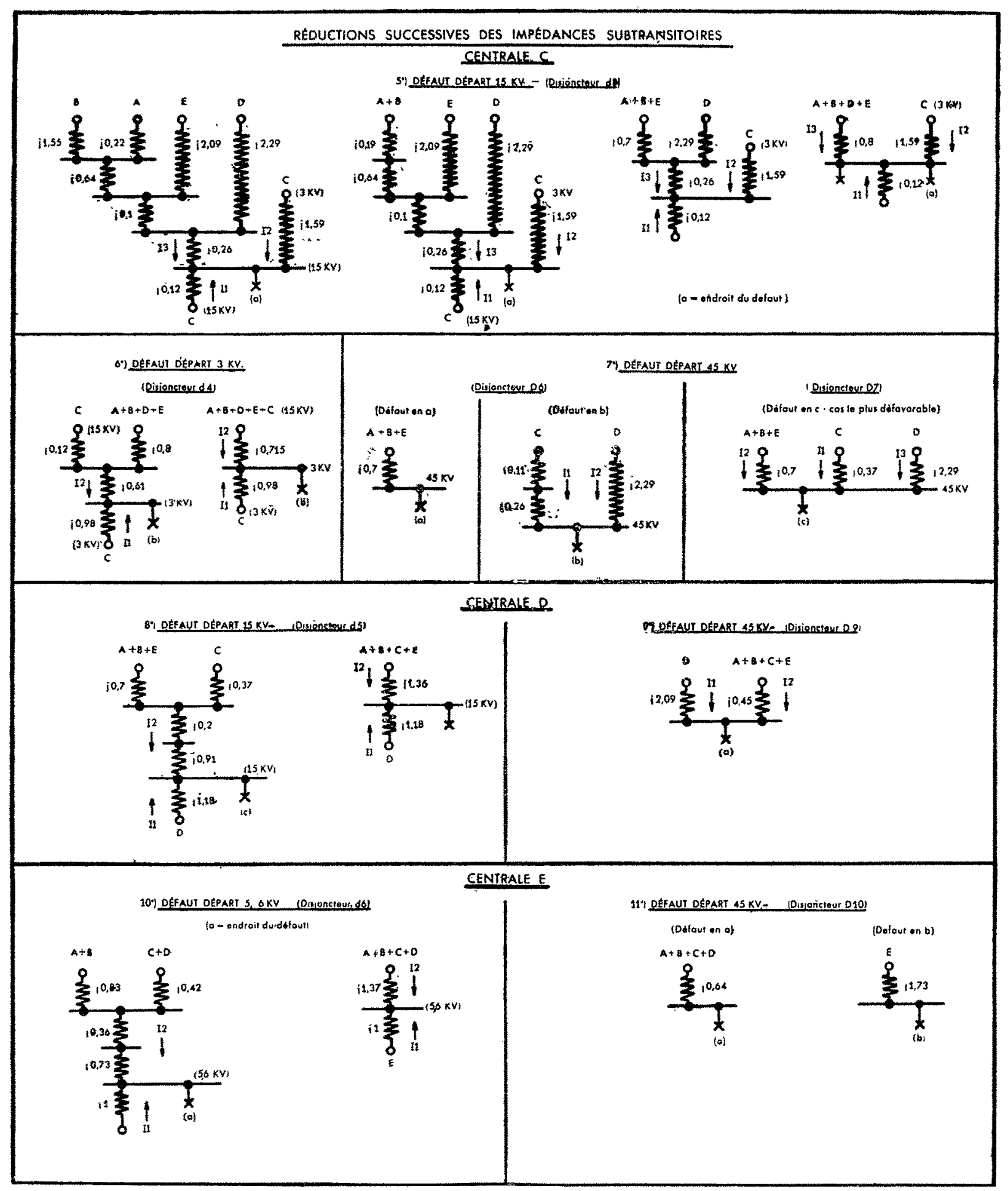

Fig. 42 bis.

c) Réductions successives des schémas des impédances.

Lors d'un défaut en un point quelconque du réseau, les diverses centrales débitent sur ce défaut.

Il faut donc, partant de chaque point de défaut, faire les réductions successives des impédances en remontant vers les centrales, afin d'obtenir l'impédance simple équivalente à l'ensemble des centrales.

Le tableau $n^{\circ} 42$ donne un exemple des réductions successives à opérer : cet exemple est traité pour les valeurs subtransitoires des réactances des alternateurs.

Le même tableau est à faire pour les valeurs transitoires et permanentes. 
L'impédance réduite permet alors de calculer le courant de c.c. aux différents instants :

\begin{tabular}{|c|c|c|c|c|c|}
\hline \multirow{2}{*}{ Centrale } & \multirow{2}{*}{ Endroit du défaut } & \multirow{2}{*}{$\begin{array}{c}\text { Disjonctour } \\
\text { Intéressé }\end{array}$} & \multicolumn{3}{|c|}{ Valeur du courant de c. $c$. } \\
\hline & & & subtr. & trans. & perm. \\
\hline A & $\begin{array}{cc}\text { départ } 10 \mathrm{kV} & \\
\text { départs } 45 \mathrm{kV} & (a) \\
& (b) \\
》 & (c) \\
》 & (d) \\
& (e) \\
& (f) \\
\end{array}$ & $\begin{array}{l}d_{1} \\
\mathrm{D}_{4} \\
\mathrm{D}_{1} \\
\mathrm{D}_{2} \\
\mathrm{D}_{2} \\
\mathrm{D}_{2}^{*} \\
\mathrm{D}_{3}\end{array}$ & $\begin{array}{r}37.450 \\
7.250 \\
6.700 \\
14.400 \\
10.150 \\
21.650 \\
24.550 \\
\end{array}$ & $\begin{array}{r}27.950 \\
6.200 \\
5.250 \\
12.100 \\
9150 \\
18.750 \\
21.250\end{array}$ & $\begin{array}{r}9.600 \\
2.230 \\
3.900 \\
5.600 \\
5.050 \\
9.400 \\
10.700\end{array}$ \\
\hline B & 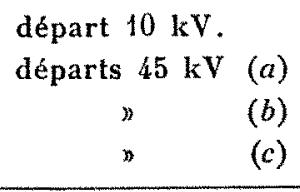 & $\begin{array}{l}d_{2} \\
\mathrm{D}_{4} \\
\mathrm{D}_{4} \\
\mathrm{D}_{5} \\
\end{array}$ & $\begin{array}{r}22.600 \\
5.950 \\
8.500 \\
11.400\end{array}$ & $\begin{array}{r}16.600 \\
5.550 \\
7.000 \\
9.600\end{array}$ & $\begin{array}{l}5.600 \\
3.500 \\
2.400 \\
4.450 \\
\end{array}$ \\
\hline C & $\begin{array}{l}\text { départ } 15 \mathrm{kV} \\
\text { départ } 3 \mathrm{kV} \\
\text { départs } 45 \mathrm{kV} \quad(\text { b) } \\
\qquad \quad(c)\end{array}$ & $\begin{array}{l}d_{3} \\
d_{4} \\
\mathrm{D}_{6}\end{array}$ & $\begin{array}{l}61.300 \\
14.600 \\
18.900 \\
27.500\end{array}$ & $\begin{array}{l}42.000 \\
12.000 \\
16.000 \\
23.900\end{array}$ & $\begin{array}{r}13.000 \\
6.350 \\
6.700 \\
11.300\end{array}$ \\
\hline $\mathrm{D}$ & $\begin{array}{l}\text { départ } 15 \mathrm{kV} \\
\text { départs } 45 \mathrm{kV}(a)\end{array}$ & $\begin{array}{l}d_{5} \\
\mathrm{D}_{9}\end{array}$ & $\begin{array}{r}9.550 \\
16400\end{array}$ & $\begin{array}{r}7.700 \\
14.650\end{array}$ & $\begin{array}{l}4.200 \\
7.600\end{array}$ \\
\hline E & $\begin{array}{l}\text { départ } 5,6 \mathrm{kV} \\
\text { départs } 45 \mathrm{kV} \quad(a)\end{array}$ & $\begin{array}{l}d_{6} \\
D_{10}\end{array}$ & $\begin{array}{r}10.460 \\
9.500\end{array}$ & $\begin{array}{l}8.900 \\
8.800\end{array}$ & $\begin{array}{l}5.500 \\
5.500\end{array}$ \\
\hline
\end{tabular}

Ces valeurs permettent de tracer la courbe de variation du courant qui intéresse chaque disjoncteur en fonction du temips (voir figure 43 pour la centrale A).
On remarquera l'allure différente de ces courbes suivant le disjoncleur considéré ; ceci provenant des valeurs différentes des impédances externes interposées entre les alternaźeurs et le point de défaut.

On ne peut donc, à priori, déterminer un rapport constant entre la valeur du courant de c. c. à l'instant initial ou après 0,1 sec. et la valeur de ce courant après $0,2 \mathrm{sec}$.

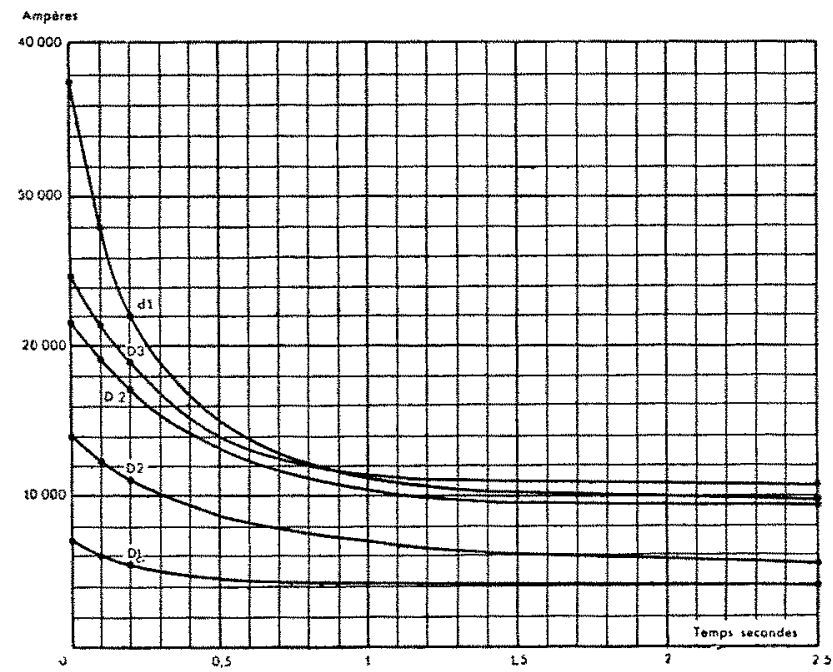

Fig. 43

On lira sur ces courbes le courant de c. c. après 0,2 seconde ; ces courants exprimés en kVA après multiplication par le facteur $10,5 \vee \overline{3}$ expriment la puissance de coupure pour une tension de rétablissement égale à la tension normale.

La figure 44 indique en regard de chaque disjoncteur sa puissance de coupure déterminée par les calculs précédents. Fille donne finalement le seul résultat lutile à retenir.

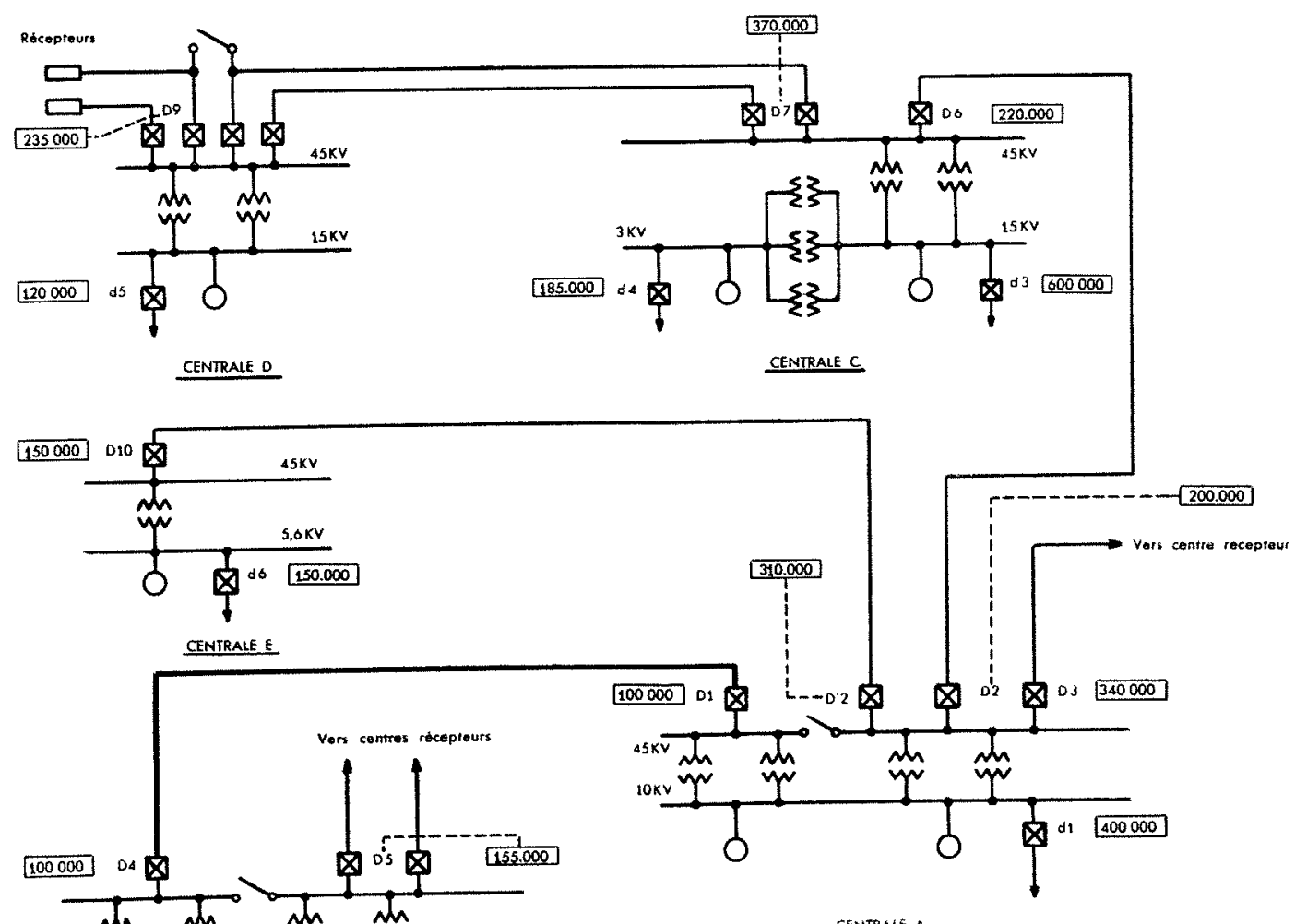

CENTRALE A

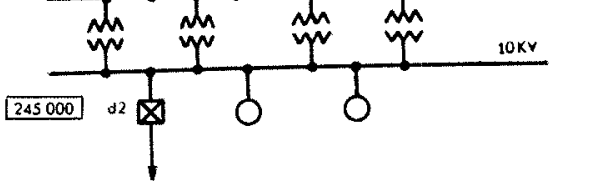

CENTRALE B. 


\section{Remarque :}

Pour les disjoncteurs de la centrale A, les courbes de lit figure 43 permettent de calculer la puissance de coupure en tenant compte de la tension de rétablissement vraie :

\begin{tabular}{|c|c|c|}
\hline \multirow{2}{*}{ Disjoncteur } & \multicolumn{2}{|c|}{$\begin{array}{c}\text { Puissance de coupure avec tension } \\
\text { de rétablissement }\end{array}$} \\
\cline { 2 - 3 } & égale à Un & \multicolumn{1}{|c|}{ calculée } \\
\hline $\mathrm{D}_{1}$ & 100.000 & 80.000 \\
$\mathrm{D}_{2}$ & 200.000 & 160.000 \\
$\mathrm{D}_{2}^{\prime}$ & 310.000 & 246.000 \\
$\mathrm{D}_{3}$ & 340.000 & 260.000 \\
$d_{1}$ & 400.000 & 235.000 \\
\hline
\end{tabular}

VII. - LA LIMITATION DES GOURANTS DE G. G. DANS UN RESEAU DU TYPE PRECEDENT, PAR LIAISON DE 2 GENERATELRS A TRAVERS UNE REAGTANCE.

Sectionnement des centrales, figure 45.

Soit une centrale de $\mathrm{N}$ alternaleurs reliés à un jeu de barres commun mn d'où partent des feeders alimentant des trans-

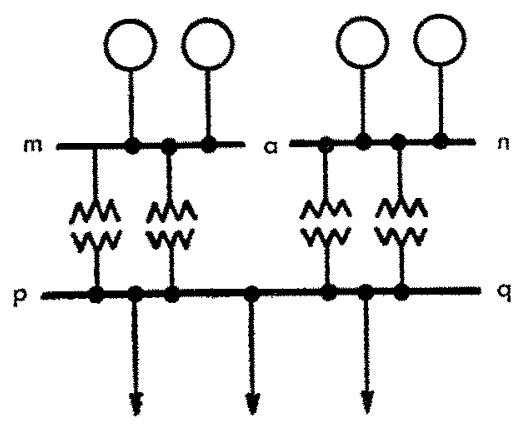

Fig. 45

formateurs élévateurs ou abaisseurs dont les secondaires sont réunis à un jeu de barres commun. Scctionnons le jeu de barres en $a$ en deux tranches dont chacune comporte alors $\frac{\mathrm{N}}{\mathrm{z}}$ machines. Ce sectionnement conserve le bénéfice de linterconnexion, car les deux tranchıs sont synchionisées à traver's la réactance des transformateurs ; et le courant de count-circuit intéressant les disjoncteurs placés sur les barres de la centrale est considérablement réduit.

Réactances de liaison.

a) Soit une centrale de $\mathrm{N}$ alternateurs reliés ì un jeu de barres commun $m n$ d'où partent des feeders radiaux.

Sectionnons comme précédemment la centrale en deux tranches et relions ces deux tranches par une réactance (fig. 46).

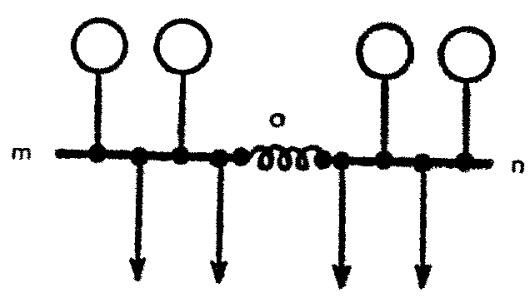

Fig. 46

Le courant de court-circuil intéressant les disjoncteurs placés sur les départs sera considérablement réduit.

b) Soit unc centrale $\mathrm{C}$ que l'on décide d interconnecter avec un réseau puissant $R$.

Pour évitor que la liaison au réseau $R$ n'augmente considémablement le court-circuit intéressant les disjoncteurs de

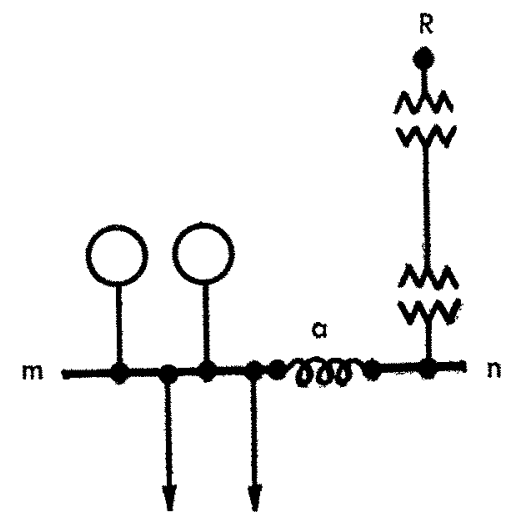

Fig. 47

départ $\mathrm{D}$, on sectionne en $a$ le jeu de barres, et on relie les 2 tranches par une réactance (fig. 47 ).

\section{Remarque.}

Lorsqu'on réunil deux parties génératrices d'un réseau à twavers une réactance, quelques problèmes particuliers sont ì considérer :

$\left.1^{\circ}\right)$ On ne peut synchroniser sans précautions à travers une réactance que deux centres générateurs voisins l'un de l'autre (quelques dizaines de $\mathrm{Km}$ ). Sinon la stabilité du système interconnecté peut être compromise : un calcul de vérification doit ctre fait.

$2^{\circ}$ ) Un avantage du couplage par réactance est que, en cas de c. c., la répercussion du c. c. sur les tensions des centres générateurs séparés du défaut par la réactance, est moindre.

$\left.3^{\circ}\right)$ Lorsque deux alternateurs de même tension sont réunis cntre cux par une réactance, tout échange de puissance réaclive entre eux est impossible.

$\left.4^{\circ}\right)$ Les réaclances introduisent des différences de phases entre les tensions, qui peuvent rendre impossibles certains bouclages.
R. L.-B, et G. F. 